\title{
Experimental Study and Nonlinear Dynamic Analysis of Time-periodic Micro Chaotic Mixers
}

\author{
Yi-Kuen Lee ${ }^{1}$, Chiang Shih $^{2}$, Patrick Tabeling ${ }^{3}$ and Chih-Ming $\mathrm{Ho}^{4}$ \\ ${ }^{1}$ Department of Mechanical Engineering \\ The Hong Kong University of Science and Technology, Kowloon, Hong Kong \\ ${ }^{2}$ Department of Mechanical Engineering \\ Florida State University, Tallahassee, FL 32310, USA \\ ${ }^{3} \mathrm{MMN}$, ESPCI, 10, rue Vauquelin, 75005 Paris, France \\ ${ }^{4}$ Department of Mechanical and Aerospace Engineering \\ University of California, Los Angeles, CA 90095, USA
}

\begin{abstract}
The efficiency of MEMS-based time-periodic micro chaotic mixers was experimentally and theoretically investigated in this study. A time-periodic flow perturbation was realized using digitallycontrolled solenoid valves to alternately activate a source and sink, acting together as a pair, with different driving frequencies. Working fluids with and without fluorescent dye were used in the micro mixing experiments. The spatiotemporal variation of the mixing concentration during the mixing process was characterized at different Strouhal numbers, ranging from 0.03 to 0.74 , under fluorescence microscopy. A simple kinematical model for the micro mixer was used to demonstrate the presence of chaotic mixing using different methods. The specific stretching rate, Lyapunov exponent, as well as local bifurcation and Poincare section analyses were used to identify the emergence of chaos. Two different numerical methods were employed to verify that the maximum Lyapunov exponent was positive in the proposed micro mixer model. A simplified analytical analysis of the effect of Strouhal number was presented. Kolmogorov-Arnold-Mose (KAM) curves, which are mixing barriers, were also found in Poincaré sections. From a comparative study of the experimental results and theoretical analysis, a Finite-Time Lyapunov exponent (FTLE) was shown to be a more practical mixing index compared to the classical Lyapunov exponent because the time spent in mixing is the main concern in practical applications, such as bio-medical diagnosis. In addition, the FTLE takes into account both fluid stretching in terms of the stretching rate and fluid folding in terms of curvature.
\end{abstract}

Keywords: chaotic mixing, Lyapunov exponent, micro mixer, Poincaré section, KAM curves Y.-K. Lee, et al., "Experimental Study and Nonlinear Dynamic Analysis of Time-periodic Micro Chaotic Mixers,” Journal of Fluid Mechanics, Vol. 575, pp.425-448, 2007.

http://dx.doi.org/10.1017/S0022112006004289 


\section{Introduction}

Micro total analysis systems (MicroTAS) and bio-micro electromechanical systems (bio-MEMS) have received much attention in industrial and academic research institutions because of their potential functionality in a variety of biological applications (Burn et al., 1998; Figeys \& Pinto, 2000; Stroock et al., 2002; Grayson et al., 2004; Stone et al., 2004). Due to their minute size and many other advantages, these micro devices are very promising for manipulating, analysing and sensing ultra small amounts of bio-samples. Depending on the types of bio-molecules (e.g., DNA, enzymes, antibodies) that are being handled, these devices must have the capability to provide efficient fluidic manipulations, such as fluid delivery, mixing, separation, and filtration.

One important fluidic manipulation is the mixing of biological fluids. This is because many biological processes, e.g., DNA purification, polymer chain reaction (PCR), and DNA hybridisation, require effective mixing to function properly. In addition, it is very common to link a DNA solution to gene expression, molecular diagnostics of diseases and so on. One way to achieve this linking is to mix a DNA solution with DNA probes and examine their reaction. Mixing in conventional laboratories is usually performed by either manually shaking, tapping the test tube followed by centrifuging, or mixing using a stirrer (Evensen et al., 1998). However, these conventional mixing methods are difficult, if not impossible, to implement in miniaturized systems. Therefore, the pursuit of novel mechanisms for effective mixing is critical for the development of bio-MEMS devices.

Traditionally, turbulent enhancement is the most effective process to achieve efficient mixing. For example, Regenfuss et al. (1985) and Bökenkamp et al. (1998) applied turbulent-jet and multiple T-shaped turbulent flows, respectively, to study fast chemical reactions at millimeter-sized scales. Nonetheless, it is much more difficult to generate 
turbulence at microscales due to the higher viscous dissipation at small Reynolds numbers (Raynal \& Gence, 1997).

However, laminar mixing through molecular diffusion is usually too slow and therefore impractical in most cases. Miyake et al. (1993) designed a double-layered micro mixer, in which 400-micron-diameter nozzles were fabricated to provide micro-plumes, which could increase the surface contact area for faster mixing. Evensen et al. (1998) designed a mixer driven by a piezo-ceramic actuator such that fluids, e.g., $\lambda$-DNA, were perturbed periodically in a glass capillary. They reported no apparent shear breakage of the DNA after mixing by testing with agarose gel electrophoresis. Bökenkamp et al. (1998) fabricated a silicon mixer for liquid phase chemical reactions over short durations of $110 \mathrm{~ms}$. Volpert et al. (1999) reported on an actively controlled micro mixer using six branched microchannels perpendicular to the main microchannel. Mixing inside the main microchannel was enhanced by introducing periodic perturbations through the side microchannels. Their numerical simulation showed that the effectiveness of this active micro mixer increased if the temporal waveforms of the perturbations are properly controlled. Unfortunately, they did not report the mixing indices, such as the Lyapunov exponent (LE) and Poincaré section, which are the most commonly used tools in the identification of chaos. Okkels \& Tabeling (2004) described the mixing in a simple cross-shaped micro mixer with time-periodic transverse flow in terms of a new phenomenon - spatial-temporal resonance. A detailed review of micro mixers designed during recent years was undertaken by Nguyen and $\mathrm{Wu}$ (2005). In particular, mixing by chaotic advection is considered to be one of the most promising techniques for mixing in microfluidic devices (Ottino \& Wiggins, 2004).

Aref was among the first researchers to use the term chaotic advection (Aref, 1984) to describe mixing achieved in a laminar flow at a low Reynolds number by comparing this phenomenon with turbulent mixing (Aref, 2002). It is well known that chaotic mixing can 
occur at any Reynolds number and that it is the degree of the spatial-temporal complexity of the flow that determines whether the advected particle paths are chaotic, not the force balance in the momentum equation (Jones, 1988).

In this paper we report on the fabrication and characterization of a time-periodic micro chaotic mixer, which was studied under fluorescence video microscopy. A 2D kinematic model of the micro chaotic mixer (Niu \& Lee 2003a) was used to conduct a parametric study of the mixing enhancement. Using different methods, the mixing enhancement could be studied in detail. Several techniques, including Poincaré section, Lyapunov exponent, and local bifurcation analyses, were used to explore the existence of chaos. In addition, a Finite-Time Lyapunov exponent (FTLE) (Pierrehumbert, 1991; Muzzio, 1991; Ottino et al., 1992; Tang \& Boozer, 1996; Thiffeault, 2004) was introduced as a mixing index for the evaluation of mixing performance of our micro mixer.

\section{Experimental apparatus and micro mixing measuring techniques}

\subsection{Fabrication of the micro mixer}

The micro mixers investigated in this study were continuous mixing systems that could be easily integrated in line with other devices to form a fully functional lab-on-a-chip system. We selected time-periodic pressure perturbations to initiate chaotic motions in order to rapidly homogenize the initially nonuniformly distributed concentration fields. The micro mixer chips were made at the UCLA Nanoelectronics Research Facility. They were fabricated using MEMS technology, including SU-8 photolithography, deep reactive ion etching (DRIE), anodic bonding, as well as other micro fabrication techniques. Crosssectional diagrams illustrating the fabrication process are shown in Figure 1. The micro mixers were fabricated on a four-inch double-sided polished silicon wafer. The following fabrication sequence was used to produce the micro mixers. First, the process was started by 
applying photolithography with SU-8 photoresist (Microchem Corp, MA, USA) as the mask for DRIE (Model SLR-770, PlasmaTherm, FL, USA) of the silicon. Second, a dummy silicon wafer was glued to the back of the first wafer by applying AZ5214 photoresist at 120 ${ }^{\circ} \mathrm{C}$ for about 5 minutes. The wafer was then etched through using the DRIE technique with SU-8 photoresist as the mask. Third, the remaining SU-8 photoresist and the dummy wafer were removed by rinsing three times consecutively in fresh strong piranha solutions $\left(\mathrm{H}_{2} \mathrm{SO}_{4}\right.$ : $\mathrm{H}_{2} \mathrm{O}_{2}=1: 1$ ). Fourth, the device was sealed by attaching the processed silicon wafer to a $1 / 8$ inch-thick Pyrex glass plate, using anodic bonding. Six through-holes were drilled on the Pyrex glass (Model 8476K321, McMaster Inc., CA, USA) to provide the fluid inlets and outlet. A thin piece of Pyrex glass $(\sim 200 \mu \mathrm{m}$, Specialty Glass Products Inc., PA, USA) bonded on the other side of the mixer was used as an optical window for flow visualization purposes. Finally, 1/16-inch barbed tubing adapters (Model 5116K16, McMaster Inc.) were machined and glued to the inlets/outlets on the thick glass layer in order to provide the necessary flow connection through Tygon ${ }^{\mathrm{TM}}$ tubing (Model 5554K91, McMaster Inc.).

As shown in Figure 2, a micro mixer with a 400- $\mu$ m-deep main channel width of 100 $\mu \mathrm{m}$ and two $50-\mu \mathrm{m}$-wide branch channels was fabricated. The micro mixer was designed so that two streams of different fluids, controlled individually by two external flow devices, could be directed through the two branch channels and merged in the main channel. This will be described in more detail in the next section.

\subsection{Experimental apparatus and characterization of the micro mixer}

The experimental setup for both the operation and characterization of the micro mixer chip is shown in Figure 3. Two digitally-controlled syringe pumps (Model 44I/W, Harvard Apparatus, MA, USA) were used to individually regulate the flow rates of the two inlets into the main channel (Figure 2). To clearly identify the mixing enhancement, Rhodamine 6G fluorescent dye (Fisher Scientific, CA, USA) was used to label the working fluid in one of the 
branch channels. As a result of the mixing, the relative fluorescent intensity of the main channel fluid stream varied due to changes in its local dye concentration; this was used as a quantitative measure of the mixing efficiency.

In addition to the micro flow visualization, it is useful to quantify the mixing efficiency. There are many ways to evaluate mixing efficiency, e.g., the residence time distribution (Levenspiel, 1972), the distribution of striation thickness (Khakhar et al., 1986), lobe dynamics (Wiggins, 1992; Beigie, et al., 1994), entropy (D’Alessandro et al., 1999) and a multiscale measure for mixing - the Mix-Norm (Mathew et al., 2005). Liu et al. (2000) evaluated the mixing of a passive three-dimensional serpentine micro mixer by considering the uniformity of the intensity of the fluid flow inside the microchannel. The uniformity was quantified by calculating the experimental mixing index $I_{E}$ by considering the deviation of the pixel intensity $C$ from its maximum intensity value in an image field such as that shown in Figure $4 b$ :

$$
I_{E}=1-\frac{1}{\bar{C}} \sqrt{\frac{\sum\left(C\left(x_{2}\right)-\bar{C}\right)^{2}}{N}},
$$

where $N$ is the total number of pixels and $\bar{C}$ is the averaged pixel intensity of the concentration function of $x_{2}, C\left(x_{2}\right)$,

$$
\bar{C}=\sum C\left(x_{2}\right) / N
$$

If ideal mixing takes place in the micro mixer, the mixed concentration is uniform and $C$ is a constant; the mixing index $I_{E}$ should assume a value of one since the root mean square integral becomes zero. If there is no mixing between the two fluid streams, $C$ is a step-like function and the mixing index is equal to zero.

To set up the main channel flow, two digital syringe pumps, each equipped with 10 $\mathrm{mL}$ glass syringes (Fisher Scientific), were used to pump de-ionized (DI) water and a DI water solution of Rhodamine $6 \mathrm{G}$ dye, respectively, into the two inlets of the micro mixer. As 
shown in Figure 3, to activate the flow control, computer-interfaced solenoid valves (threeway HDI Model LHDA 0531115H, The Lee Company, CT, USA), which could be turned on and off at specified frequencies, were integrated with the control ports of the micro mixer. The control signal for the solenoid valves had a periodic square waveform with variable frequencies, generated by a PIO-12 digital input/output card (Keithley Instruments Inc., OH, USA) interfaced to a personal computer. When connected to a high-pressure source and/or a low-pressure sink, these valves could be activated to provide pulsatile pressure perturbations to the main channel flow at desired frequencies and amplitudes. The high-pressure source was regulated by a compressed air tank with a two-stage pressure regulator, while the lowpressure sink was controlled by a rotary vane vacuum pump (Edwards E2M18).

To prevent air bubbles from getting into the microchannel, two transparent Plexiglas reservoirs were added between the micro mixer and the solenoid valves (Figure 3). Extra care was taken to ensure that the majority of the trapped air was collected and removed inside these reservoirs. In order to adjust the strength of the source and sink to achieve the desired perturbation, pinch-tight tube clamps (Model 5330K14, McMaster Inc.) were used on the 1/16-inch-diameter Tygon $^{\mathrm{TM}}$ tubing (Model 5554K91, McMaster Inc.) connecting the vacuum pump and the compressed air tank to the two Plexiglas reservoirs.

\subsection{Micro flow visualization}

An epi-fluorescence Olympus IX70 microscope was used to visualize the micro mixing flow. The fluorescent light emitted from the fluorescent dye-labelled liquid flow could be efficiently separated from the light scattered from the channel walls and other impurities in the testing liquids. The flow behaviour inside the main channels after the two branch-flow streams merged was recorded on videotape using a CCD camera (Model TM745, Pulnix Inc., CA, USA). The video was then digitised by a video capture interface card (TARGA 1000, 
Truevision Inc., CA, USA) with the image resolution of $648 \times 486$ pixels (NTSC) and analysed by using an in-house Matlab $^{\mathrm{TM}}$ digital image-processing program. The mixing enhancement could be further quantified by examining the normalized fluorescence intensity distribution of the fluid across the main channel at the $A A$ cross section as shown in Fig. $4 b$ and using Equation (1).

The micro mixer was tested with two low-diffusivity working fluids consisting of a glycerine (AC41098-5000, Fisher Scientific) water solution with and without Rhodamine 6G dye. Without time-periodic perturbations in the mid-stream, the downstream profile showed little change compared with its upstream counterpart. The slight broadening of the concentration profile in the $x_{2}$ direction could be attributed to the molecular diffusion of the fluorescent dye. If the average velocity in the main microchannel, controlled by a digital syringe pump, is $V_{0}$, then the diffusion constant of Rhodamine 6G $D$ can be estimated by measuring the broadening width at the downstream location $x_{1}$ (Lee et al., 2000).

In Figure $4 b$, the micro mixer with the time-periodic perturbation in the mid-stream had a Strouhal number of 0.056 (frequency $f=0.80 \mathrm{~Hz}$ ). Here, the Strouhal number is defined as $\mathrm{St}=f W_{s} / V_{0} . V_{0}$ is the average velocity of the main microchannel, $f$ is the frequency of perturbations (which was varied) and $W_{s}$ is the width of the side channel, respectively. In an active micro mixer, St represents the ratio between the residence time of a species and the time period of its disturbance (Nguyen $\& \mathrm{Wu}, 2005$ ). At $\operatorname{Re}=2.4 \times 10^{-4}$, the main channel in the micro mixer showed a repetitive fluid interface folding pattern right after the side channel, as shown in Figure $4 b$. This lobe-like fold pattern greatly increased the surface of the contact area of the two fluids to promote mixing. At the end of the downstream location, molecular diffusion gradually blurred this lobe-like folding structure, signifying a more effective mixing process. 
In addition, the controlled fluid interface pattern was examined using pressure perturbations at different driving frequencies and amplitudes. At low frequencies and amplitudes, the interface showed a strong wavy pattern. If the driving frequency increased, the amplitude of the wavy pattern decreases accordingly. However, for the same perturbation frequency, the folding level increased as the amplitude increased. In Section 4, we will examine numerically how a micro mixer with time-period perturbations in the side channels can cause 'chaotic mixing' by computing the Lyapunov exponent and plotting a Poincaré section.

We recorded the periodic perturbation of the two fluids in the micro mixer and examined the evolution of the fluid interface during one complete period ( $T=1 \mathrm{~s})$, as shown in Figure 5. Pseudo colour was applied to these micrographs via digital image-processing to enhance the fluid interface between the two fluids. In the first three frames $(t=0,0.1 T$ and $0.2 T$ ), the fluids at the intersection of the main channel and side channel were pushed upward in the side channel (positive $x_{2}$ direction). This created a folding of the fluids. In the following frames, the stretched fluid blob was pulled in the negative $x_{2}$ direction. The fluid blob was also forced to move into the main channel, following the main flow stream. Because the velocity profile in the main channel was parabolic, the fluid blob was deformed nonuniformly across the main channel width, resulting in fluid stretching. Under the timeperiodic flow perturbations in the side channel, the micro mixer created a series of fluid stretching and folding patterns that are essential for increasing the fluid interfaces, thus promoting good mixing.

Next, we adjusted the driving frequency of the micro mixer to study the effect of frequency on mixing efficiency. For each case, a 30-s video of the flow field at a downstream location was recorded. The experimental mixing index $I_{E}$ for each frame was calculated, and the time-averaged mixing index as a function of Strouhal number is shown in Figure 6. From 
this figure, the optimal mixing for this micro mixer occurred at a Strouhal number close to 0.25 .

\section{Modelling and analysis of the micro chaotic mixer}

In order to gain a better understanding of how the micro mixer works, a kinematical model will be developed in this section. Lyapunov exponent, local bifurcation and Poincaré section analyses will be applied to study the non-linear dynamic behaviour of this micro mixer model.

\subsection{Kinematic model for the micro mixer}

Since the flow velocity in our micro mixer was extremely low (typically, the Reynolds number $<<1$ ), the Navier-Stokes equations can be linearized to equations representative of Stokes' flow. Under Stokes' flow approximation, the velocity at the intersection of the main and branch channels may be approximated as a linear superposition of the main flow plus the perturbed flow. Note that although the velocity field is linear, the governing equations for the fluid particle trajectory are non-linear. A detailed chaotic dynamic analysis will be discussed later in Section 4.

As shown in Figure $7 a$, we assumed that the main channel, with a width $W_{m}$, flowed from left to right, and that there was one side channel at the origin of the coordinates with a width $W_{s}$. According to the local flow behaviour, we could divide the velocity field into four regions: I, II, III, and IV, as shown in Figure $7 a$. The velocity profiles, both in the main (Regions I and III) and side channels (Region IV) far from the intersection, were assumed to be parabolic. The main flow had a constant flow rate while the side channel carried a timedependent component. At the intersection between the side and main channels (Region II), the resultant velocity was the linear sum of the two components from both channels. For Regions I and III, there was only one non-zero velocity component in the $x_{1}$ direction: 


$$
\frac{d x_{1}}{d t}=V_{0}\left(1-\left(X_{2} / W_{m}\right)^{2}\right), \frac{d x_{2}}{d t}=0 \text { for Regions I \& III, }
$$

where $V_{o}$ is the maximum velocity in the main channel.

Note that $X_{1}$ and $X_{2}$ are the material coordinates. The motion $\mathbf{x}=\mathbf{x}(\mathbf{X}, t)$ denotes the position vector $\mathbf{x}=\left(x_{1}, x_{2}\right)$ occupied by the material particle $\mathbf{X}=\left(X_{1}, X_{2}\right)$ at time $t$ (Eringen, 1967) along the streamwise $\left(x_{1}\right)$ and the transverse directions $\left(x_{2}\right)$. This notation is useful for computing deformation rate tensors, the differential line stretch, and the stretching rate for quantitative mixing analysis (Ottino, 1989). For the side channel far from the intersection, such as Region IV, we assumed that the velocity was only in the $x_{2}$ direction. The velocity amplitude in Region IV changed periodically with respect to time at a driving frequency $\omega$ :

$$
\frac{d x_{1}}{d t}=0, \frac{d x_{2}}{d t}=V_{P}\left(1-\left(X_{1} / W_{s}\right)^{2}\right) \cos (\omega t) \text { for Region IV, }
$$

where $V_{p}$ is the maximum velocity amplitude in the side channel.

The velocity field in Region II was also assumed to be the linear superposition of those fields in Regions I and IV:

$$
\frac{d x_{1}}{d t}=V_{o}\left(1-\left(X_{2} / W_{m}\right)^{2}\right), \frac{d x_{2}}{d t}=V_{P}\left(1-\left(X_{1} / W_{s}\right)^{2}\right) \cos (\omega t) \text { for Region II. }
$$

Although this is an approximated velocity field, this assumption is not expected to affect the qualitative results substantially, as pointed out by Volpert et al. (1999) in a similar geometry and by the CFD verification in our previous work (Lee et al., 2000; Niu \& Lee, 2003a). Once the velocity field is prescribed, the particle trajectories can be readily calculated by integrating the governing ordinary differential equations. Note that effect of diffusion has not been factored into our model due to its negligible contribution to the overall flow behaviour as evident from experimental observations. The distribution of a total of 24,000 colour-coded particles moving in the micro mixer $\left(V_{p} / V_{0}=0.5, \mathrm{St}=0.637\right)$, is shown in Figure $7 b$. A time-periodic stretching and folding process can be clearly observed in this 
flow pattern, consistent with the micro flow visualization results using a low-diffusivity working fluid (Figure 4b).

The driving frequency, $\omega(=2 \pi f)$, and the relative strength of the side-channel perturbation, $\left(V_{p} / V_{\mathrm{o}}\right)$, in the dynamic system equations are both important parameters that govern the interface pattern of the two mixing fluid streams. If $V_{o}$ were fixed while $V_{p}$ and $\omega$ were varied gradually in the simulation of the fluid interface, the result will lead to very rich diversity of flow patterns. Figure $7 b$ represents the shape of a fluid interface released at the centre of the main channel upstream from the channel intersection after passing through the mixing region (where the side channel is located). At a small amplitude, the interface underwent oscillations as it passed through the mixer. This is classified as the wavy regime. The wave amplitude increases with the perturbing amplitude and leads to the so-called 'strong waves' regime. Finally, a chaotic-like regime appeared beyond the threshold amplitude, when the wave periodically hit a corner of the channel intersection. A more detailed analysis can be found in Tabeling et al. (2004).

The traditional infinite-time Lyapunov exponent, which indicates the chaotic mixing, is mathematically defined for fluid properties that are calculated at a time approaching infinity, i.e., the side-channels in the micro mixer need to be repeated indefinitely. Obviously this is not a useful quantity in practical microfluidic applications. The governing equation for the micro mixer, Equation 2, can be modified to allow for infinite downstream side channels. This can be easily implemented in a microfluidic device and in a computer program by assigning side cross channels with normalized widths of 2 at $x_{1}=4 n-1(n=1,2,3, \ldots)$. This approach has been successfully implemented to simulate a micro mixer with normalized channel dimensions. However, in order to examine the sensitivity of the geometric design (main and side channel widths) to the mixing, additional computer code was introduced for 
the velocity field with a main channel width $\left(W_{\mathrm{m}}\right)$, side channel width $\left(W_{\mathrm{s}}\right)$, and distance between side channels ( $p c h)$ as follows:

1. If $\operatorname{Abs}\left(x_{2}\right) \leq W_{m n} / 2, \dot{x}_{1}=V_{o}\left(1-\left(x_{2} /\left(W_{m} / 2\right)\right)^{2}\right)$, otherwise $\dot{x}_{1}=0$.

2. Set $x_{p}=p c h^{*}$ Integer $\operatorname{part}\left(x_{1} / p c h\right)$.

3. If $x \leq\left(x_{\mathrm{p}}+W_{\mathrm{s}}\right)$, Set $x_{c}=x_{p}+W_{s} / 2$ and

$$
\dot{x}_{2}=V_{p}\left(1-\left[\left(x_{1}-x_{c}\right) /\left(W_{s} / 2\right)\right]^{2}\right) \cos (\omega t) \text {. }
$$

4. If $x>\left(x_{p}+W_{s}\right)$, Set $\dot{x}_{2}=0$.

Simulations of the deformation of two fluid blobs in the micro mixer with periodic side channels at different Strouhal numbers were conducted with the above modified model. As shown in Figure 8, the original fluid blobs were transformed into much smaller fluid segments at $\mathrm{St}=0.32$ than those at $\mathrm{St}=0.24$ or 0.95 . However, these types of simulations were very time-consuming. It was impractical to conduct comprehensive investigation of mixing effectiveness. Therefore, the parametric study of the effect of the Strouhal number on the mixing performance will be discussed using a different approach in Section 4.

\subsection{Specific stretching rate as a measure of the mixing effectiveness: an analytical solution}

As we have discussed in previous sections, the surface contact area (fluid interface or material line in $2 \mathrm{D}$ flow) plays an important role in the mixing process. The time-periodic forcing in the side channel greatly enhances the mixing process by increasing the total contact area at the fluid interface. Therefore, one way to characterize the mixing enhancement in a two-dimensional micro flow field is to calculate the increase of the individual fluid interface lines. However, as pointed out by Franjione \& Ottino (1987), direct numerical tracking of the interface presents formidable computational problems, even in simple two-dimensional chaotic flows. Tracking a realistic three-dimensional flow would be even more challenging. 
However, a Lyapunov exponent analysis has been shown to be an effective alternative approach to characterizing the mixing effectiveness because the Lyapunov exponent $\lambda_{i}$ can be physically interpreted as the long-term average of the specific stretching rate (Khakhar et al., 1986; Ottino, 1989).

A dynamic system with $n$ degrees of freedom has at most $n$ different Lyapunov exponents $\lambda_{i}(i=1,2, \ldots, n)$. If $|d \mathbf{X}|$ represents the length of an infinitesimal fluid element vector with initial conditions $d \mathbf{X}$ around $\mathbf{X}$ and orientation $\mathbf{M}=d \mathbf{X} /|d \mathbf{X}|$, and its length at time $t$ is $|d \mathbf{x}|$, the Lyapunov exponents $\lambda_{i}$ are defined as

$$
\lambda_{i}(\mathbf{X}, \mathbf{M})=\lim _{\substack{t \rightarrow \infty \\|d \mathbf{X}| \rightarrow 0}}\left[\frac{1}{t} \ln \left(\frac{|d \mathbf{x}|}{|d \mathbf{X}|}\right)\right]
$$

where $\mathbf{X}$ and $\mathbf{M}=\left\{M_{i}\right\}$ are the position and orientation vectors of the initial fluid element. From chaotic dynamics, it is well known that at least one Lyapunov exponent in a chaotic system will be positive. The stretching efficiency $e(\mathbf{X}, \mathbf{M})$, also considered as a theoretical mixing index (a normalized Lyapunov exponent; Ottino, 1989), is defined as

$$
e(\mathbf{X}, \mathbf{M}) \equiv \frac{d_{i j} d_{i j} M_{i} M_{j}}{\left(d_{i j} d_{i j}\right)^{1 / 2}},
$$

where $i$ and $j$ are the dummy indices for deformation rate tensor $\left(d_{i j}\right)$ summation.

If a flow field can be described analytically, such as 2D parabolic channel flow, we can easily derive the theoretical mixing index, $e(\mathbf{X}, \mathbf{M})$. In practice, it is more efficient to compute the line stretch first and then differentiate the line stretch with respect to time. From a study of continuum mechanics (Eringen, 1967), the square of the line stretch $s$ for an infinitesimal fluid element with an orientation vector $\mathbf{M}$ is

$$
s^{2}=C_{i j} M_{i} M_{j}
$$

where $C_{i j}$ is the right Cauchy-Green strain tensor. This tensor can simply be found by using the following formula: 


$$
\mathbf{C}=\mathbf{F}^{\mathrm{T}} \mathbf{F},
$$

(6)

where $\mathbf{F}=\left\{\partial x_{i} / \partial X_{j}\right\}$ is the deformation gradient tensor.

For 2-D parabolic flow, the solution of the position of a fluid particle as a function of its original position, $\mathbf{X}=\left(X_{1}, X_{2}\right)$, is

$$
x_{1}=V_{o}\left(1-X_{2}^{2}\right) t+X_{1}, \quad x_{2}=X_{2} .
$$

Therefore, the corresponding right Cauchy-Green strain tensor is

$$
\mathbf{C}=\mathbf{F}^{T} \mathbf{F}=\left(\begin{array}{cc}
1 & -2 V_{o} t X_{2} \\
-2 V_{o} t X_{2} & 1+4 V_{o}^{2} t^{2} X_{2}^{2}
\end{array}\right)
$$

The square of the line stretch for a fluid element with an original orientation vector $\mathbf{M}=\left(M_{1}\right.$, $\left.M_{2}\right)$ is therefore

$$
s^{2}=M_{1}^{2}+M_{2}^{2}-4 M_{1} M_{2} V_{o} t X_{2}+4\left(M_{2} V_{o} t X_{2}\right)^{2} .
$$

Differentiating Equation (9) with respect to time and dividing by $\left(d_{i j} d_{i j}\right)^{1 / 2}$, we can find the theoretical mixing efficiency as follows:

$$
\begin{gathered}
e(\mathbf{X}, \mathbf{M})=\frac{1}{\sqrt{2} X_{2} V_{o}} \frac{4\left(M_{2} V_{o} X_{2}\right)^{2} t-2 M_{1} M_{2} V_{o} X_{2}}{M_{1}^{2}+M_{2}^{2}-4 M_{1} M_{2} V_{o} t X_{2}+4\left(M_{2} V_{o} t X_{2}\right)^{2}} \\
=\frac{2 \sqrt{2} M_{2}^{2} X_{2} V_{o} t-\sqrt{2} M_{1} M_{2}}{M_{1}^{2}+M_{2}^{2}-4 M_{1} M_{2} V_{o} t X_{2}+4\left(M_{2} V_{o} t X_{2}\right)^{2}}
\end{gathered},
$$

where $\left\{d_{i j}\right\}=\left(\begin{array}{cc}0 & -V_{o} X_{2} \\ -V_{o} X_{2} & 0\end{array}\right), \quad d_{i j} d_{i j}=2 d_{12}^{2}=2\left(V_{o} X_{2}\right)^{2}$.

For $t=0, e(\mathbf{X}, \mathbf{M})$ can be simplified as

$$
e(\mathbf{X}, \mathbf{M})=\frac{-\sqrt{2} M_{1} M_{2}}{M_{1}^{2}+M_{2}^{2}}
$$

The maximum $e(\mathbf{X}, \mathbf{M})$ is obtained for a fluid line element with the following orientation vector: 


$$
M_{1}=\frac{-1}{\sqrt{2}} \quad M_{2}=\frac{1}{\sqrt{2}}
$$

This is consistent with a previous paper (Khakhar et al., 1986). As $t \rightarrow \infty$, we find that the mixing index, $e(\mathbf{X}, \mathbf{M})$, of the 2-D parabolic flow decreases as $1 / t$,

$$
e(\mathbf{X}, \mathbf{M}) \rightarrow \frac{2 \sqrt{2} M_{2}^{2} X_{2} V_{o} t}{4\left(M_{2} V_{o} t X_{2}\right)^{2}}=\frac{\sqrt{2}}{2 V_{o} X_{2} t} \sim \frac{1}{t}
$$

Therefore, 2-D parabolic channel flow without any perturbation does not mix well. This gives us the mathematical foundation necessary to introduce an external control to enhance mixing. Recently, complementary to our analysis of a micro mixer model, Voth et al. (2002) also used the Cauchy-Green strain tensor to evaluate the Finite-Time Lyapunov Exponent field and distribution of the experimental data of a 2D flow produced by density stratification and time-periodic magnetic forcing. Obviously, this approach will be a very useful tool for the comparative study of chaotic mixing research between theory and experiment.

\subsection{Dimensionless temporal parameter for chaotic mixing}

When considering chaotic mixing at the macro-scale level, the contribution of molecular diffusion is usually negligible. However, at the micro-scale level, mixing due to diffusion may not be neglected. Yang (1994) used a simplified dimensional analysis and found that the characteristic mixing length scale and time scale for chaotic mixing with diffusion were $L_{c}=\sqrt{D / \lambda}$ and $\tau=\ln \left(L_{0} / L_{c}\right) / \lambda$, respectively, where $\lambda$ is the Lyapunov exponent, $L_{0}$ is the initial length scale, and $D$ is the fluid diffusion constant. If the characteristic length, $L_{c}$, is substituted into $\tau$, we have

$$
\tau=\frac{\ln \left(L_{o} / \sqrt{D / \lambda}\right)}{\lambda}=\frac{2 \ln \left(L_{o} / \sqrt{D / \lambda}\right)}{2 \lambda}=\frac{\ln \left(\lambda L_{o}^{2} / D\right)}{2 \lambda} .
$$

However, an analysis of an advection-convection equation in a natural Lagrangian coordinate system (Tang \& Boozer, 1996) illustrated a dimensionless parameter for the chaotic mixing: 


$$
\Omega=\frac{\lambda L_{o}^{2}}{D}=\frac{L_{o}^{2} / D}{1 / \lambda}=\frac{t_{D}}{t_{C}}
$$

where the diffusive mixing time, $t_{D}$, is $L_{o}^{2} / D$ and the chaotic mixing time, $t_{C}$, is $1 / \lambda$. In fact, after rearranging the above equation, we can show that this dimensionless parameter is exactly the Peclet number (Levenspiel, 1972):

$$
\Omega=\frac{L_{o}^{2} / D}{1 / \lambda}=\frac{L_{0}}{t_{C}} \frac{L_{0}}{D}=\frac{V_{c} L_{0}}{D}=\mathrm{Pe} .
$$

Therefore, the chaotic mixing time, $t_{C}$, can be simply expressed as

$$
t_{C}=\frac{\ln (\mathrm{Pe})}{2 \lambda} \sim \ln \left(L_{0}\right)
$$

This mixing time, $t_{C}$, is proportional to the logarithm of the characteristic length scale, $L_{0}$. Therefore, we can use an estimation of the chaotic mixing time as a function of Peclet number and Lyapunov exponent to design a micro mixer for different types of fluids. In comparison to the diffusive mixing time $\left(t_{D} \sim L_{o}^{2}\right.$ ), which is proportional to the second power of the characteristic length scale, chaotic mixing is much more efficient.

\section{Nonlinear dynamic analysis of the micro chaotic mixer model: numerical approach}

Due to the large non-linear perturbation terms in Equation (2), it is impossible to follow the same approach presented in previous section to find the Lyapunov exponents. Therefore, we will use numerical methods in this section. Since a Lyapunov exponent analysis is not trivial, two different methods that can be used to find the Lyapunov exponent(s) will be considered in the following sections.

\subsection{Lyapunov exponent analysis, method I: direct method}

The actual calculation of the Lyapunov exponent is not trivial, because by definition, the Lyapunov exponent is a long-term average of the increase in the specific length. A practical way to find the Lyapunov exponent is to split the ratio of final position to initial 
position, $\|\mathbf{X}\| /\|\mathbf{x}\|$, into a large number of small time-steps. The time-step can be set to 0.001 (Sprott, 2003) so that the Runge-Kutta numerical integration method can be readily used. The initial distance between the two particles is about three to four orders of magnitude larger than the minimum precision of a computer. For example, for a typical double-precision floating point in a Pentium PC with a 64-bit processor, we can have 15-digit precision. Thus the initial separation can be set at about $10^{-12}$ to $10^{-11}$.

First, a computer code developed in Microsoft Visual C++ was applied to calculate the Lyapunov exponents of well-known chaotic systems, e.g., the Lorenz attractor and the Rosseler attractor (Tufillaro et al., 1992) as listed in Table 1 (the total number of time steps is $10^{8}$ ). All the relative errors between our numerical results and reference values were less than $0.3 \%$.

Next, the computer code was used to calculate the Lyapunov exponents of the micro mixer model described in Section 3.1 for different frequencies $\left(\mathrm{St}=f W_{0} / V_{0}\right)$ and a fixed amplitude $\left(V_{p} / V_{0}=1\right)$. The variables $W_{m}$ and $W_{\mathrm{s}}$ were set to be $W_{0}$. The Lyapunov exponent of the micro mixer required about 100 million time-steps to converge while those of the Lorenz attractor only required 1 million time-steps according to the detailed convergence study in our previous work (Niu \& Lee 2003a). The maximum Lyapunov exponent as a function of the Strouhal number, calculated using method I, is shown in Figure 9. Note that method I can only be used to calculate the maximum Lyapunov exponent, which is the most important one to characterize chaotic mixing. However, method II in Section 4.2 can be used to calculate all of the Lyapunov exponents.

\subsection{Lyapunov exponent analysis, method II: modified QR method}

The Lyapunov exponents have been shown to be a generalization of the eigenvalues at an equilibrium point (Parker \& Chua, 1989): 


$$
\lambda_{i} \equiv \lim _{t \rightarrow \infty} \frac{1}{t} \ln \left|m_{i}(t)\right|, \quad i=1, \cdots, n
$$

where $m_{i}(t)$ are the characteristic multipliers of the variational equation associated with the solution, $\varphi_{t}\left(\boldsymbol{x}_{o}, t\right)$, to the original dynamic system $\dot{\mathbf{x}}=f(\mathbf{x}, t), \mathbf{x}\left(t_{o}\right)=\mathbf{x}_{o}$,

$$
\frac{d \boldsymbol{\Phi}}{d t}=D_{x} f(\mathbf{x}) \boldsymbol{\Phi}, \quad \boldsymbol{\Phi}_{o}=\mathbf{I}
$$

where $D_{x} f(\mathbf{x})$ is a tangent map, a matrix-valued function from the vector field, $f(x)$ and $\Phi_{\mathbf{t}}\left(\mathbf{x}_{\mathbf{0}}, t\right) \equiv D_{\mathbf{x} 0} \phi_{t}\left(\mathbf{x}_{\mathbf{0}}, t_{o}\right)$. Using the above definition, there are a series of published papers (Benettin et al., 1980; Dieci \& van Vleck, 1995; von Bremen, 1997) that discuss how to efficiently calculate the Lyapunov exponents. For example, the Lyapunov exponents can be computed by calculating sequence of eigenvalues for the tangent map, $D_{x} f(\mathbf{x})$, at different times. An efficient method, the QR-based algorithm for factorizing (an essential step in computing $\lambda_{i}$ ) the system matrix was recently reported (von Bremen et al., 1997). We developed a computer program in Matlab using this new method and found three Lyapunov exponents for the micro mixer model: one zero, one positive, and one negative. The maximum calculated Lyapunov exponent was consistent with those determined by method I described in previous section. The occasional large discrepancies in the solid curve in Figure 9 are due to relative small number of time steps $(20,000)$ used in Method II. Note that method I can be used to find the maximum Lyapunov exponent, while Method II can be used to find all the Lyapunov exponents. The maximum Lyapunov exponents as a function of the Strouhal number were compared using these two methods, as shown in Figure 9. The optimal Strouhal number for mixing in this Lyapunov exponent curve was $\sim 1$.

However, this St value is not consistent with the experimental results (Figure 4). According to the existing literature (Tang \& Boozer, 1996) and our previous analysis (Niu \& 
Lee, 2003b), we found that a Finite-Time Lyapunov exponent (FTLE), $\lambda(\tau)$ is a better criterion to characterize the mixing efficiency of micro mixers,

$$
\lambda(\tau)=\lim _{|d \mathbf{X}| \rightarrow 0}\left[\frac{1}{\tau} \ln \left(\frac{d \mathbf{x}(\tau)}{d \mathbf{X}}\right)\right],
$$

The FTLE will converge to an infinite-time LE, Equation (3), as the time increases to infinity. But the variation of the FTLE depends on the initial points and time-steps. For the 2-D or 3D conservative systems that are often investigated, the spatially averaged (or mean)

FTLE,$<\lambda>$, can be expressed as (Tang \& Boozer, 1996)

$$
\langle\lambda\rangle=\langle\tilde{\lambda}(\xi)\rangle / t+\langle f(\xi, t)\rangle / \sqrt{t}+\lambda,
$$

where first term depends only on the initial conditions, while the second term also depends on time. Both terms tend to zero as $t$ approaches infinity. Therefore, the FTLE converges quickly to an infinite-time LE even though the second term can induce some small fluctuations according to the dynamic properties of the system. Figure 10 shows the mean FTLE distribution of the active micro mixer in the $\omega-V_{p}$ parameter space (Niu $\&$ Lee, 2003b), which can be used to select the optimal parameters, such as the perturbation amplitude $V_{p}$ and the operating frequency $\omega$. An array of 50 by 50 points in one square area of the main microchannel at the inlet were used as the initial conditions for the calculation of the mean FTLE in Fig. 10. The side of the square area is the length that the fluid in the main microchannel moves at $V_{0}(=1)$ for one period, $1 / f$. The total number of time steps of integration is 10,000 for each of the initial points with a time step of 0.001 . The advantage of the FTLE analysis is that it can decrease the CPU time by more than three orders of magnitude for our micro mixer. From this figure, the optimum Strouhal number is $\sim 0.5$. This estimation is better than those based on the infinite-time Lyapunov exponent analysis, which implies that the FTLE is a better mixing index for our micro mixer. The reason for this result is that the FTLE accounts for both fluid stretching in terms of the stretching rate and fluid 
folding in terms of curvature (Thiffeault, 2004), while an infinite-time Lyapunov exponent involves only fluid stretching.

\subsection{Local bifurcation and Finite-Time Lyapunov exponent analyses}

The existence of saddle points in the flow field is important. The saddle point in a 2D velocity field is a point in between the attraction and repulsion of forces. An analysis of a saddle point can be very useful in understanding chaotic dynamic systems. If the cycle time of the time-periodic forcing matches the residence time of the fluid particle (the particle can easily travel to the saddle point), then the mixing can be greatly enhanced. This is due to the frequent coincidence of the saddle point and the transverse homoclinic or heteroclinic point (Tufillaro et al. 1992). Chaos theory has shown that the existence of a single homoclinic or heteroclinic point forces the existence of an infinite number of such points. This also gives rise to a homoclinic tangle (Tabor, 1989). In addition, the optimal driving frequency can be found by analysing the motion of a fluid particle travelling from the main channel to the saddle point.

In order to clarify the locations of saddle points or similar critical points in our micro mixer model, we performed a local eigenvalue analysis as follows:

$$
\dot{\mathbf{x}}=f(\mathbf{x})=\mathbf{f}\left(\mathbf{x}_{\mathbf{o}}\right)+\left.\frac{\partial f}{\partial \mathbf{x}}\right|_{\mathbf{x}_{\mathbf{0}}}\left(\mathbf{x}-\mathbf{x}_{\mathbf{0}}\right),
$$

where time $t$ has been replaced by $x_{3}$ for the sake of convenience. At the intersection of the main and side channels (for the sake of clarity, we set $W_{s}$ and $W_{m}$ to be 1), the Jacobian matrix $(\partial f / \partial \mathrm{x})$ is

$$
\left[\frac{\partial f(\mathbf{x})}{\partial \mathbf{x}}\right]=\left(\begin{array}{ccc}
0 & -2 V_{o} x_{2} & 0 \\
-2 x_{1} V_{p} \cos \left(x_{3}\right) & 0 & -V_{p}\left(1-x_{1}^{2}\right) \sin \left(x_{3}\right) \\
0 & 0 & 0
\end{array}\right)
$$

The eigenvalues for this matrix can be easily calculated as 


$$
0, \pm 2 \sqrt{V_{o} x_{1} x_{2} \cos \left(x_{3}\right)}
$$

As shown in Figure 11, for the region $\left(x_{1}, x_{2}\right)>0\left(x_{1,2}=1\right.$ and $\left.x_{1,2}=-1\right)$, during the time intervals $2 n \pi<\omega t<(2 n+1 / 2) \pi$ and $(2 n+3 / 2) \pi<\omega t<2(n+1) \pi(n=0,1,2, .),.\left(x_{1}, x_{2}\right)$ are saddle points, while for the region $x_{1} x_{2}<0\left(x_{1}=-1, x_{2}=1\right.$ and $\left.x_{1}=1, x_{2}=-1\right),\left(x_{1}, x_{2}\right)$ are centre points. However, during the time intervals $(2 n+1 / 2) \pi<\omega t<(2 n+3 / 2) \pi$, the previous saddle and centre points will exchange roles with each other.

Note that the classification into centre and saddle points applies to the tangent plane of the velocity field, not to the velocity field itself. Although the velocity field is very regular at any time, the velocity gradient field with respect to $x_{1}, x_{2}$, and $x_{3}(=t)$ exhibits singular behaviour.

The optimal driving frequency for the best mixing enhancement in our proposed micro mixer was determined from the experiment in Section 2 and from the Lyapunov exponent analysis given in Section 4.2. Here, we demonstrate that the optimal frequency condition can be roughly estimated by considering the motion of the fluid particle initially at $\left(-W_{s} / 2,0\right)$ and finally at $\left(W_{s} / 2, W_{m} / 2\right)$. Since this particle is in Region II of Figure $7 a$, the governing equation for the particle trajectory is

$$
\frac{d x_{1}}{d t}=V_{o}\left(1-\left(X_{2} / W_{m}\right)^{2}\right), \quad \frac{d x_{2}}{d t}=V_{P}\left(1-\left(X_{1} / W_{s}\right)^{2}\right) \cos (\omega t)
$$

Because this set of ordinary differential equations is highly non-linear, it is difficult to find an analytical solution. Therefore, we simplified the velocity in the $x_{1}$ direction by assuming it was uniform to obtain an approximated solution:

$$
\frac{d x_{1}}{d t}=V_{o}, \quad \frac{d x_{2}}{d t}=V_{P}\left(1-\left(X_{1} / W_{s}\right)^{2}\right) \cos (\omega t),
$$

with initial conditions $x_{1}(0)=-W_{s} / 2$ and $x_{2}(0)=0$.

The solution to this simplified model can be easily found: 


$$
\begin{aligned}
& x_{1}(t)=x_{1}(0)+V_{o} t=\frac{-W_{s}}{2}+V_{o} t \\
& x_{2}(t)=x_{2}(0)+\int_{0}^{t} V_{p} \cos (\omega t) d t=\frac{V_{p}}{\omega} \sin (\omega t)
\end{aligned} .
$$

Since we imposed the final location of the particle, $\left(W_{s} / 2, W_{m} / 2\right)$, we can find the particle travel time $T_{f}$,

$$
x_{1}\left(T_{f}\right)=\frac{W_{s}}{2}=\frac{-W_{s}}{2}+V_{o} T_{f}, \quad T_{f}=\frac{W_{s}}{V_{o}},
$$

Substitution of this travel time into the $x_{2}$ solution gives

$$
\begin{gathered}
\frac{W_{m}}{2}=\frac{V_{p}}{\omega} \sin \left(\omega T_{f}\right)=\frac{V_{p}}{\omega} \sin \left(\frac{\omega W_{s}}{V_{o}}\right) \\
\mathrm{St}=\frac{f W_{m}}{V_{p}}=\frac{1}{\pi} \sin \left(2 \pi f T_{f}\right) .
\end{gathered}
$$

or simply

When $2 \pi f T_{f}=\pi / 2$ and $V_{p}=V_{0}$, the above equation can be reduced to

$$
\mathrm{St}=\frac{1}{\pi} \approx 0.32
$$

The estimated optimal driving frequency in this manner turns out to be close to the experimental result $(\mathrm{St} \sim 0.25)$ as shown in Figure 6.

\subsection{Poincaré Section Analysis}

A Poincaré section analysis is commonly used to simplify the analysis of an $n$-dimensional dynamic system by reducing it to $n-1$ dimensions (Tufillaro et al., 1992). For an integrable 2D Hamiltonian system, such as a harmonic oscillator without damping, the Poincaré section is simply a point in the phase space. Therefore, periodic systems have a finite set of points. In contrast, chaotic systems have an infinite number of points on a Poincaré section. Chaotic regions appear as a random array of dots, while regular regions are indicated by the presence 
of well-defined invariant curves in the flow. It is well known that Poincaré section analysis cannot distinguish between quasi-periodic and chaotic motion. Therefore, the Lyapunov exponent analysis was necessary to clarify this ambiguity.

For the micro mixer model, the Poincaré section method can be applied by setting an appropriate mapping. First, we set $z=\omega t$ and span the governing equation into an autonomous system. For the trajectory of any initial point, we choose the Poincaré section to be $x_{n}=n \times p c h, n=1,2, \cdots$. The trajectory will intersect these planes successively at points $P_{1}, P_{2}, \cdots, P_{n}$ and define a mapping $P_{n+1}=\Phi\left(P_{n}\right)$. With this mapping, $z$ goes to infinity as $t$ increases. Since $z$ only appears in the autonomous system as a term of a sinusoidal function, we can define a new variable, $\alpha=\bmod (z, 2 \pi)$. Substituting $\alpha$ for $z$ in the original system will not change its dynamic characteristics. Therefore we have defined a new mapping, $P_{n}\left(y_{n}, \alpha_{n}\right)$. Because the micro mixer equation is a highly non-linear system, we cannot obtain an explicit formula for this mapping. However, each point can be calculated by integrating the equation into the next Poincaré section plane (Niu \& Lee, 2003a).

Figure 12 shows Poincare sections of the chaotic mixer for two perturbations with the same operating frequency but different amplitudes. The dotted areas represent the chaotic regions. A point that starts within this area will have the possibility of ending up at any location within this area. Regions represented by circles and lines denote quasi-periodic motions or torus; particles that initiated in these regions can only move in a periodic or quasiperiodic manner. The Kolmogorov-Arnold-Mose (KAM) curves separate the periodic region from the chaotic region (Ottino, 1989; Tufillaro et al., 1992). Any trajectory of a point initiated in one attractor (the quasi-periodic area or chaotic area) cannot cross over the KAM curves to another attractor. Therefore, the KAM curves act as boundaries that prevent the mixing of fluid elements between the two types of regions. 
In Figure $12 a$, when $V_{p}=0.15$, a large portion of the elements continued to move periodically as indicated by the presence of wavy lines in the centre of the channel. Only particles close to the channel walls became chaotic. But even inside these supposedly chaotic regions, there existed small patches of periodic regions. As the perturbation amplitude increased, these patches of periodic regions eventually broke up further such that inner chaotic regions emerged from within, as shown in Figure $12 b$. However, near the centre of the channel, the size of the dominant periodic region was reduced, at first gradually. Later, it degenerated into a string of periodic islands, as shown in Figure $12 b$. This string of islands acted as a barrier that separated the upper and lower chaotic areas, preventing any exchanges between the upper and lower regions.

Finally, when $V_{p}$ increased beyond a critical value $\sim 0.42$, the string of islands will broke up into isolated periodic patches and chaotic pathways appeared between these islands. Under these circumstances, chaotic fluid elements on one side of the channel could pass along these pathways to the other side, as shown in Figure $12 b$. As $V_{p}$ increased further, this chain of isolated islands not only shrank in size but also broke into even smaller patches until they were all engulfed by the chaotic regions. From a parametric study of Poincaré sections, we showed that the best mixing was achieved when the amplitude of the side-channel perturbation was designed to be greater than 0.42 . The critical $V_{p}$ value found here is consistent with the result of the Lyapunov exponent analysis.

The stable and unstable manifolds of a hyperbolic fixed point in a nonlinear dynamic system have been shown to be an invariant sets (Ottino, 1989). A single transverse intersection between these manifolds is sufficient to produce chaotic behavior (Lichtenberg \& Lieberman, 1982). In classical chaotic mixing flows, such as a tendril-whorl flow or blinking vortex flow (Khakhar et al, 1986), the stable and unstable manifolds can be easily determined because there are analytical solutions for fixed points in these flow fields. For our micro 
mixer model, it is much more difficult to determine the stable and unstable manifolds because we do not have analytical solutions for the fixed points. The stable and unstable manifolds of a hyperbolic fixed point, i.e., $(\alpha, y)=(4.40,-0.45)$, were found at $V_{p}=0.47$ and $\omega=1$ (Figure 13). However, a comprehensive study of all the manifolds in the micro mixer model is beyond the scope of this paper.

\section{Conclusions}

A two-stream micro mixer with time-periodic perturbations in the side channels was fabricated using MEMS technology. Glycerine solutions with and without fluorescent dye were used to characterise the micro mixer chips, using fluorescence microscopy, at different frequencies (or Strouhal numbers, St). An experimental mixing index based on the standard deviation of the intensity of the pixels in the digital images was introduced to quantify the mixing process. From a time-averaged mixing index versus frequency plot, the best mixing occurred at $\mathrm{St} \sim 0.25$.

In addition, a kinematic model was constructed to track tens of thousands of fluid particles in the channel. These particles exhibited a lobe-like structure pattern after the perturbation, which was consistent with the experimental results. Lyapunov exponent, Poincaré section, and local bifurcation analyses were performed to provide a quantitative indication of the chaotic mixing in our proposed micro mixer model.

The Poincaré sections of the micro mixer were used to identify regular and chaotic regions for different perturbation amplitudes. Chaotic regions appeared as a random array of dots while regular regions were indicated by the presence of well-defined invariant curves in the flow. Since the chaotic system is usually very complicated, in addition to Poincaré section analysis, a Lyapunov exponent analysis was also used in this paper. 
The existence of chaotic mixing in the micro mixer model with time-periodic flow perturbations in the side channels was confirmed by the computation of Lyapunov exponents with two different numerical methods. From a Lyapunov exponent versus frequency plot, the best long-term mixing occurred at $\mathrm{St} \sim 1$. However, a method to achieve better mixing over a short time period is of more practical interest in microfluidic applications. In addition, a local bifurcation analysis of the micro mixer model showed that the intersections of the main and side channels were saddle point candidates. The distance between the fluid particles initially near these saddle points diverged as time increased.

In the numerical model, the FTLE was found to be a maximum at $\mathrm{St} \sim 0.5$ while the infinite-time Lyapunov exponent was a maximum at $\mathrm{St}=1$; on the other hand, in the experiment, the mixing index was found to be a maximum at $\mathrm{St}=0.25$. One may thus suggest that, in order to obtain appreciable mixing in the mixer we considered, it is preferable to optimize the FTLE rather than the infinite-time Lyapunov exponent. The former takes more completely the dynamical processes at work into account, while the latter puts emphasis on the stretching of fluid elements which may not imply mixing, as already mentioned by a number of authors (e.g. Ottino, 1989).

\section{Acknowledgements}

The authors would like to thank Prof. W. Ditto at the Georgia Institute of Technology, Dr. Daniel S. Choi at the Jet Propulsion Laboratory, Tom Lee and Steve Franz at the UCLA Nanolab and Dr. Xize Niu at HKUST for their assistance. This work is supported by the HKUST I²MS grant (Ref No: I2MS01/02.EG08) and PROCORE France/Hong Kong Joint Research Scheme (Ref No: F-HK18/05T). 


\section{References}

AREF, H. 1984 Stirring by chaotic advection. J. Fluid Mech. 143, 1-21.

Aref, H. 2002 The development of chaotic advection. Phys. Fluids 14(4), 1315-1325.

Beigie, D., Leonard, A. \& Wiggins, S. 1994 Invariant manifold templates for chaotic advection. Chaos Solitons Fractals 4, 749.

Benettin, G., Galgani, L., Giorgilli, A. \& Strelcyn, J. M. 1980 Lyapunov characteristic exponents for smooth dynamical systems and for Hamiltonian systems; a method for computing all of them. Parts I and II. Meccanica 15, 9-30.

Bökenkamp, D., Desai, A., Yang, X., Tai, Y.-C., Marzluff, E. M. \& Mayo, S. L. 1998 Microfabricated silicon mixers for submillisecond quench-flow analysis. Anal. Chem. 70, 232-236.

Burns M. A., Johnson B. N., Brahmasandra S. N., Handique K., Webster J. R., Krishnan M., Sammarco T. S., Man P. M., Jones D., Heldsinger D., Mastrangelo C. H. \& Burke D. T. 1998 An Integrated Nanoliter DNA Analysis Device. Science 282(5388), 484-487.

D’alessandro, D., Dahleh, M. \& Mezic, I. 1999 Control of mixing in fluid flow: a maximum entropy approach. IEEE T. Automat. Contr. 44, 1852-1863.

DIECI, L. \& VAN VLECK, E., 1995 Computation of a few Lyapunov exponents for continuous and discrete dynamical systems. Appl. Numer. Math., 17(3), 275-291.

Evensen, H. T., Meldrum, D. R. \& Cunningham, D. L. 1998 Automated fluid mixing in glass capillaries. Rev. Sci. Instrum. 69 (2), 519-526.

ERINGEN, A. C. 1967 Mechanics of Continua, Wiley, New York.

FigeYs, D. \& PinTO, D. 2000 Lab-on-a-chip: A revolution in biological and medical sciences. Anal. Chem. 72(9), 330A-335A.

FranjIONE, J. G. \& OtTINO, J. M. 1987 Feasibility of numerical tracking of material lines and surface in chaotic flows. Phys. Fluids. 30(12), 3641-3643.

Grayson, A. C. R., Shawgo, R. S., Johnson, A. M., Flynn, N. T., Yawen, L. I., Cima, M. J. \& LANGER, R. 2004 A BioMEMS review: MEMS technology for physiologically integrated devices. P. IEEE 92(1), 6-21.

JonEs, S. \& AREF, H. 1988 Chaotic advection in pulsed source-sink systems. Phys. Fluids 31, $469-485$.

KHAKHAR, D. V., Rising, H. \& OtTINO, J. M. 1986 Analysis of chaotic mixing in two 
model systems. J. Fluid Mech. 172, 419-451.

LeE, Y.-K., TABeling P., SHIH, C. \& Ho, C. M. 2000 Characterization of a MEMSFabricated Mixing Device. In Proceedings of ASME IMECE 2000, Orlando, FL, USA, Nov 5-10, 505-511.

LeVENSPIEL, O. 1972 Chemical Reaction Engineering, Wiley, New York.

Lichtenberg, A. J. \& Lieberman, M. A. 1982 Regular and Stochatic Motion, Springer, New York.

Liu, R. H., Stremler, M. A., Sharp, K. V., Olsen, M. G., Santiago, J. G., Adrian, R. J., Aref, H. \& BeEBE, D. J. 2000 Passive mixing in a three-dimensional serpentine microchannel. J. of MEMS. 9, 190-197.

Mathew, G., Mezić, I. \& Petzold, L. 2005 A multiscale measure for mixing. Physica D 211(1-2), 23-46.

Miyake, R., Lammerink, T. S. J., Elwenspoek, M., \& Fluitman, J. H. J. 1993 Micro mixer with fast diffusion. In Proceedings of IEEE MEMS'93, Fort Lauderdale, FL, 248253.

Muzzio, F. J., Swanson, P. D. \& OtTino, J. M. 1991 The statistics of stretching and stirring in chaotic flows. Phys. Fluids 3(5), 822-834.

Nguyen, N.-T. \& Wu, Z. 2005 Micromixers - a Review. J. Micromech. Microeng. 15, R1R16.

NIU, X. \& LEE, Y.-K. $2003 a$ Efficient spatial-temporal chaotic mixing in microchannels, $J$. Micromech. Microeng. 13(3), 454-462.

NIU, X. \& LEE, Y.-K. 2003b Finite time Lyapunov exponent for micro chaotic mixer design. In Proceedings of MEMS, ASME IMECE'03, Washington DC, Nov. 16-21 (Paper No: IMECE2003-41389).

OKKELS, F. \& TABELING, P. 2004 Spatiotemporal resonances in mixing of open viscous fluids, Phys. Rev. Lett. 92(3), 038301.

Otinno, J. M. 1989 The Kinematics of Mixing: Stretching, Chaos, and Transport, Cambridge University Press, New York.

Ottino, J. M., Muzzıo, F. J., Tjahjadi, M., Franjione, J. G., Jana, S. C. \& Kusch, H. A. 1992 Chaos, symmetry, and self-similarity: Exploiting order and disorder in mixing processes. Science 257, 754-760.

Otinno, J. M. \& Wiggins, S. 2004 Introduction: mixing in microfluidics. Phil. Trans. R. Soc. Lond A 362, 923-935. 
PARKer, T. S. \& ChUA, L. O. 1989 Practical Numerical Algorithms for Chaotic Systems. Springer, New York, 67.

PIERREHUMBert, R. T. 1991 Large-scale horizontal mixing in planetary atmospheres. Phys. Fluids A 3(5), 1250-1260.

RAYNAL, F. \& GENCE, J.-N. 1997 Energy saving in chaotic laminar mixing. Int. J. Heat Mass Transfer 40(14), 3267-3273.

Regenfuss, P., Clegg, R. M., Fulwyler, M. J., Barrantes, F. J. \& Jovin, T. M. 1985 Mixing liquids in microseconds. Rev. Sci. Instrum. 56(2), 283-290.

Sprott, J. C. 2003 Chaos and Time-Series Analysis, Oxford University Press, New York, 116-117 (also http:// sprott.physics.wisc.edu/chaos/lyapexp.htm).

Stone, H. A., Stroock, A. D. \& Ajdari, A. 2004 Engineering flows in small devices: microfluidics toward a lab-on-a-chip. Ann. Rev. of Fluid Mech. 36, 381-411.

Stroock, A. D., Dertinger, S. K. W., Ajdari, A., Mezic, I., Stone, H. A., Whitesides, G. M. 2002 Chaotic mixer for microchannels, Science. 295(5555), 647-651.

Tabeling, P. Chabert, M., Dodge, A., Jullien, M.-C. \& OkKels F. 2004 Chaotic mixing in cross-channel micromixers. Phil. Trans. R. Soc. Lond A 362, 987-1000.

TABOR, M. 1989 Chaos and Integrability in Nonlinear Dynamics: An Introduction. Wiley, New York, 145.

TANG, X. Z. \& BoOZER, A. H. 1996 Finite time Lyapunov exponent and advection-diffusion equation. Physica D 95, 283-305.

Thiffeault, J. L. 2004 Stretching and curvature of material lines in chaotic flows. Physica D 198 (3-4), 169-181.

Tufillaro, N. B., Aвbott, T. \& Reilly, J. 1992 An Experimental Approach to Nonlinear Dynamics and Chaos. Addison-Wesley, New York.

Volpert, M., Meinhart, C. D., Mezic, I., \& Dahelh, M. 1999 An actively controlled micromixer. In Proceeding of MEMS, ASME IMECE, Nashville, TN, USA, Nov., 483487.

von Bremen, H. F., Udwadia, F. E. \& Proskurowski, W. 1997 An efficient QR based method for the computation of Lyapunov exponents. Physica D 101, 1-16.

Voth, G. A., Haller, G. \& Gollub, J. P. 2002 Experimental measurements of stretching fields in fluid mixing. Phys. Rev. Lett. 88(25), 254501.

WigGINS, S. 1992 Chaotic transport in dynamical systems. Springer-Verlag, New York.

YANG, H. 1994 On the relative importance between chaotic mixing and diffusion. Physics Letters A 185(2), 191-195. 


\section{List of figures}

Figure 1. Cross-sectional diagrams of the micro fabrication process flow for the micro mixer, (a) photolithography using SU-8 photoresist, (b) dummy wafer gluing, (c) DRIE etching, (d) double-sided anodic bonding, and (e) barbed adapter gluing.

Figure 2. Schematic diagram of the micro mixer.

Figure 3. Schematic diagram of the experimental setup for the micro mixer characterization. Figure 4.(a) Schematics of a simple cross-channel micro mixer and (b) generation of a lobelike fold structure between the two fluids (glycerine with and without Rhodamine $6 \mathrm{G}$ dye) by periodic pressure perturbation at the side channel at $\mathrm{Re}=2.4 \times 10^{-4}$ and $f$ $=0.80 \mathrm{~Hz}$.

Figure 5. Pseudo-colour enhanced micrographs showing the generation of stretching and folding of the fluids (glycerine solution with and without Rhodamine 6G dye) in the micro mixer at different stages during one period $(T=1 \mathrm{~s})$ at $\operatorname{Re}=2.4 \times 10^{-4}$ and $\mathrm{St}=0.08$.

Figure 6. Time-averaged experimental mixing index as a function of the Strouhal number, St, for the micro mixer.

Figure 7. (a) The velocity fields in the micro mixer model divided into Regions, I, II, III, and $\mathrm{IV}$, and (b) the generation of a lobe-like structure by the periodic perturbation of the two-stream fluids to increase the surface contact area. $X_{1}$ and $X_{2}$ are the material coordinates $\left(V_{p} / V_{0}=0.5, \mathrm{St}=0.637\right)$.

Figure 8. Simulations of mixing of two fluid blobs in the time-periodic micro mixer at different driving frequencies at $t^{*}\left(=t / t_{0}\right)=13.3\left(t_{0}=d / V_{0}\right.$,avg $\left.=3\right)$ : (a) initial fluid blobs at $t^{*}=0$; (b) $\mathrm{St}=0.24, t^{*}=13.3$; (c) $\mathrm{St}=0.32, t^{*}=13.3$; and (d) $\mathrm{St}=0.95, t^{*}=13.3$.

Figure 9. Comparison of the Lyapunov exponent of the micro mixer model as a function of the Strouhal number using two different methods: method I (dotted), method II (solid line with the error bars). The error bar denotes the standard deviation of the 10,000 Lyapunov exponents along the trajectory before the final time step. Initial condition $(x, y)=(0,0)$, Initial orientation $=(1,0), V_{p}=1.5$.

Figure 10. Contour plot of the mean Finite-Time Lypaunov exponent of the micro mixer model on the $\omega-V_{p}$ plane $\left(V_{o}=1\right)$.

Figure 11. Eigenvalue analysis of the micro mixer model at the intersection of the main and side channels: (a) when $2 n \pi<\omega t<(2 n+1 / 2) \pi$ and $(2 n+3 / 2) \pi<\omega t<2(n+1)$; 
and (b) when $(2 n+1 / 2) \pi<\omega t<(2 n+3 / 2) \pi,(n=0,1,2, .$.$) .$

Figure 12. Poincaré sections of the micro mixer model at different conditions: (a) the two types of KAM curves formed at $V_{p}=0.15$; and (b) the train of periodic islands that appeared at $V_{p}=0.43$.

Figure 13. Stable (grey line) and unstable manifold (black line) of a hyperbolic fixed point on the Poincaré sections of the micro mixer model at $V_{p}=0.471$ and $\omega=1$. 


\begin{tabular}{|l|l|l|l|l|}
\hline & Parameters & Reference & Method I & Rel. Error \\
\hline Lorenz attractor & $\sigma=10, r=28, b=8 / 3$ & 0.906 & 0.904 & $0.22 \%$ \\
\hline Lorenz attractor & $\sigma=16, r=45.92, b=4$ & 1.50 & 1.504 & $0.27 \%$ \\
\hline Rössler attractor & $a=b=0.2, c=5.7$ & 0.0714 & 0.0711 & $0.04 \%$ \\
\hline
\end{tabular}

Table 1. Verification of the Lyapunov exponent computations calculated by method I using reference values from Tufillaro et al. (1992). 


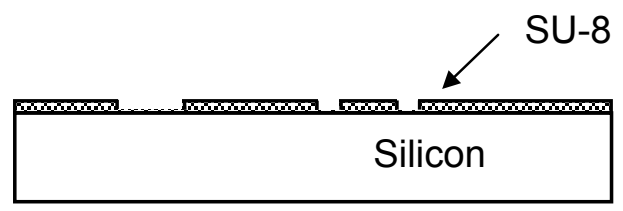

(a)

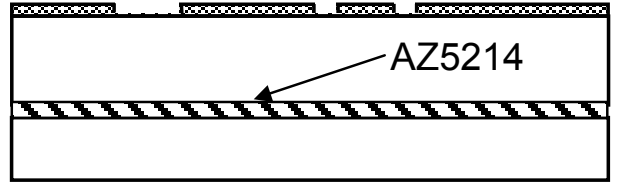

(b)

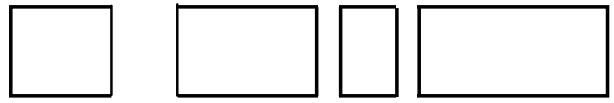

(c)

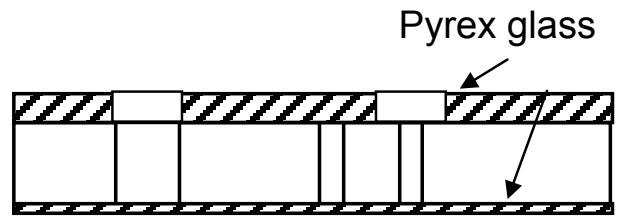

(d)

Barbed adapter

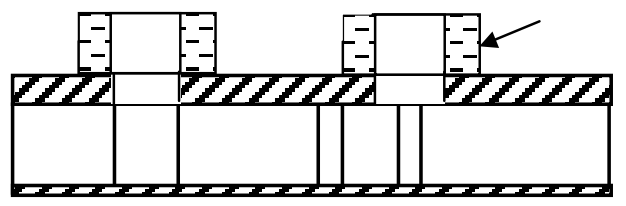

(e)

Figure 1. Cross sectional diagrams of the micro fabrication process flow for the micro mixer, (a) photolithography using SU-8 photoresist, (b) dummy wafer gluing, (c) DRIE etching, (d) double-sided anodic bonding, and (e) barbed adapter gluing. 


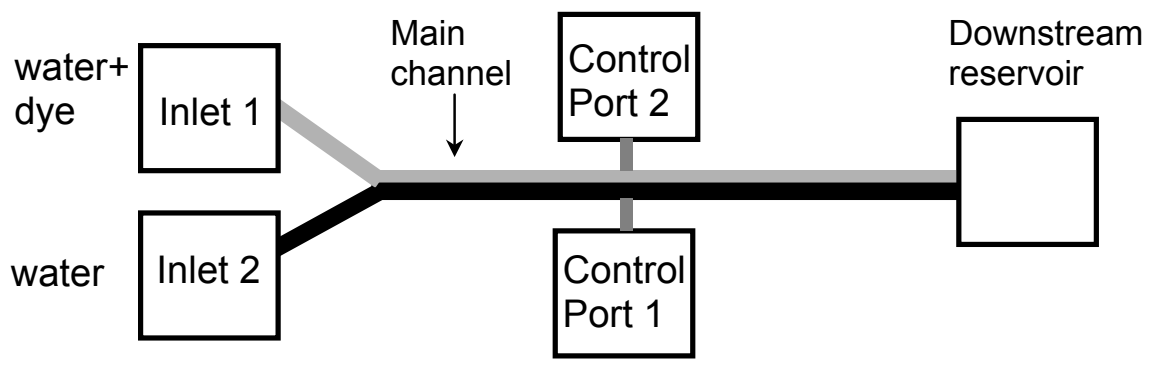

Figure 2. Schematic diagram of the micro mixer. 


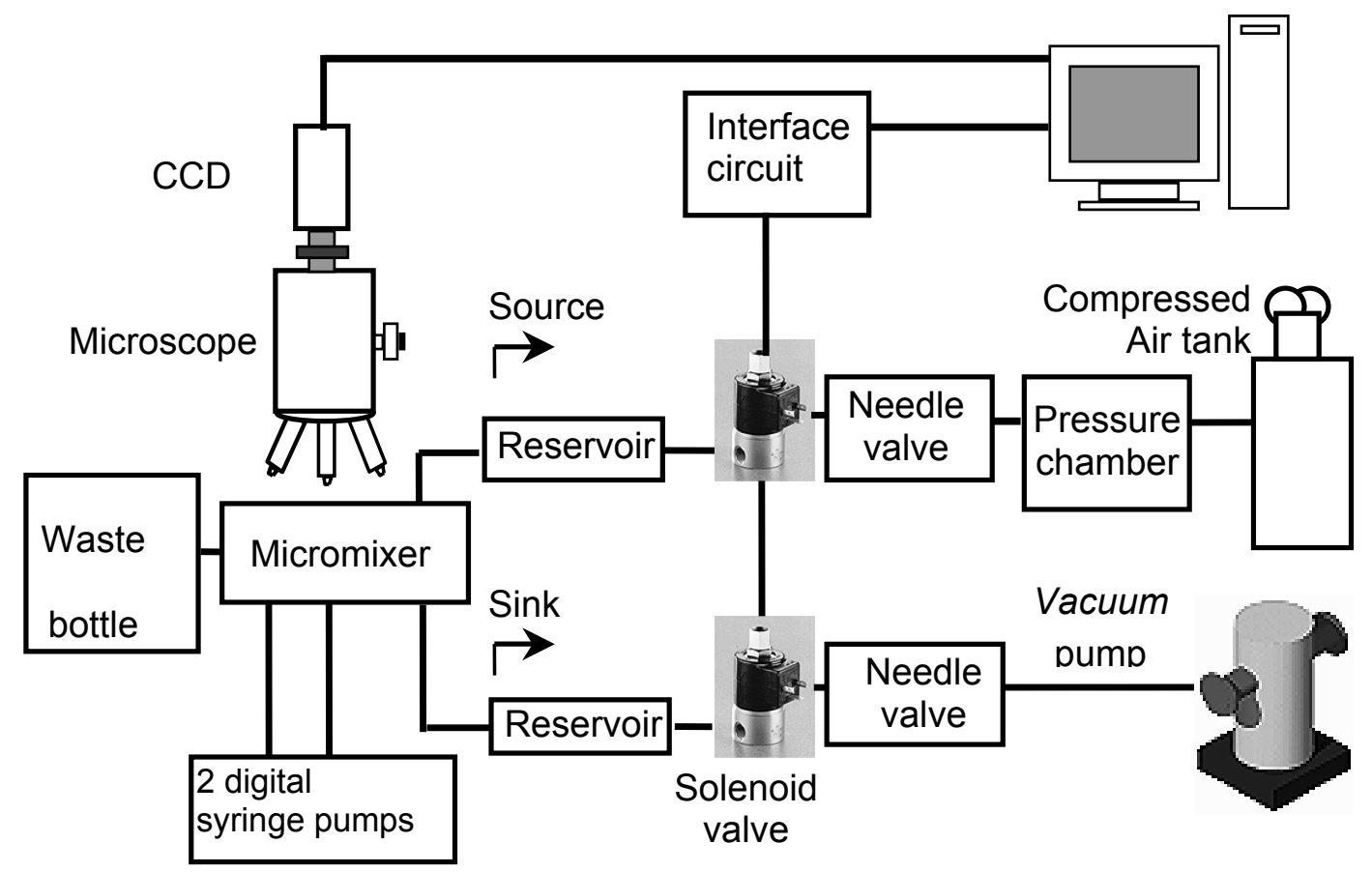

Figure 3. Schematic diagram of the experimental setup for the micro mixer characterization. 


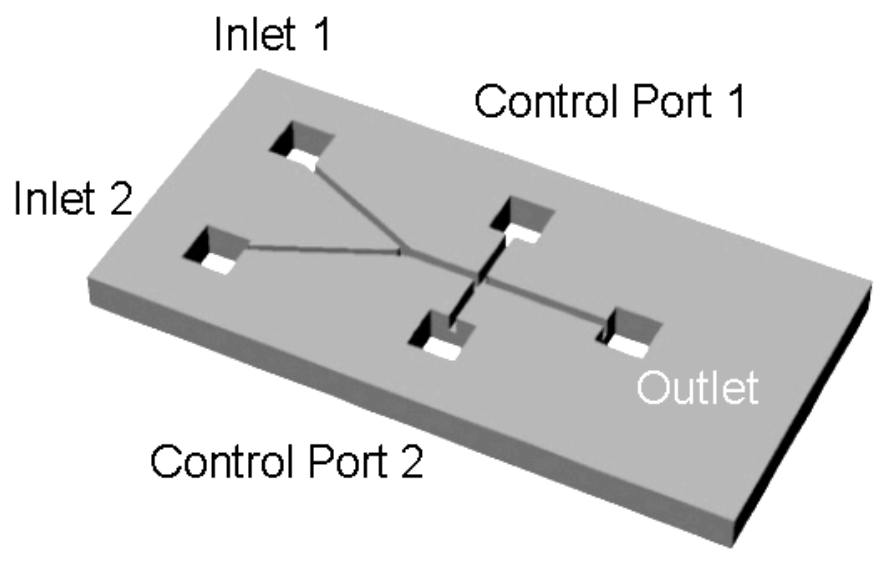

(a)

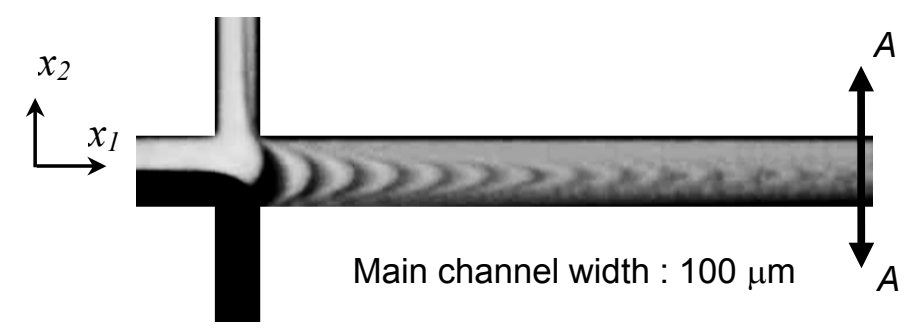

(b)

Figure 4. (a) Schematics of a simple cross-channel micro mixer, and (b) generation of a lobe-like fold structure between the two fluids (glycerine with and without Rhodamine $6 \mathrm{G}$ dye) by periodic pressure perturbation at the side channel at $\operatorname{Re}=2.4 \times 10^{-4}$ and $f=$ $0.80 \mathrm{~Hz}$. 

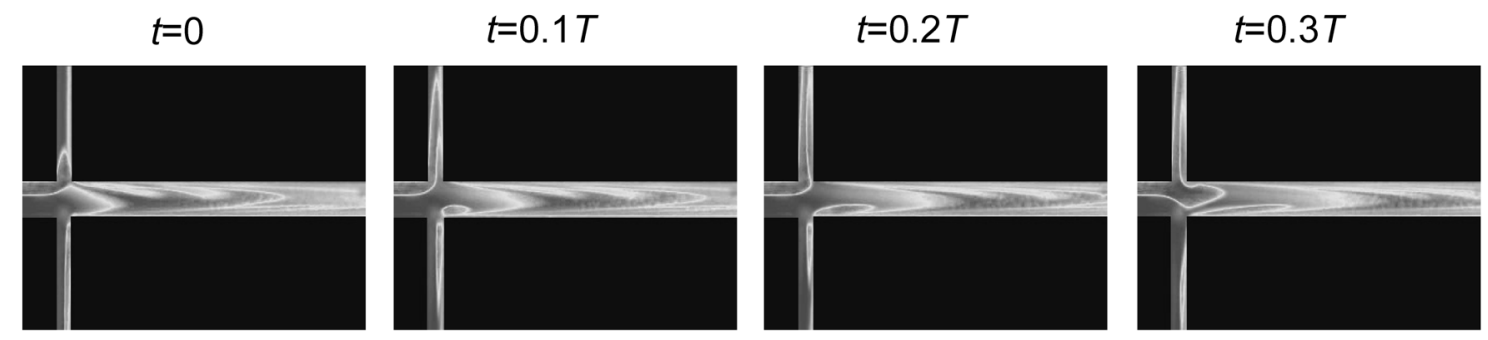

$x_{2}$
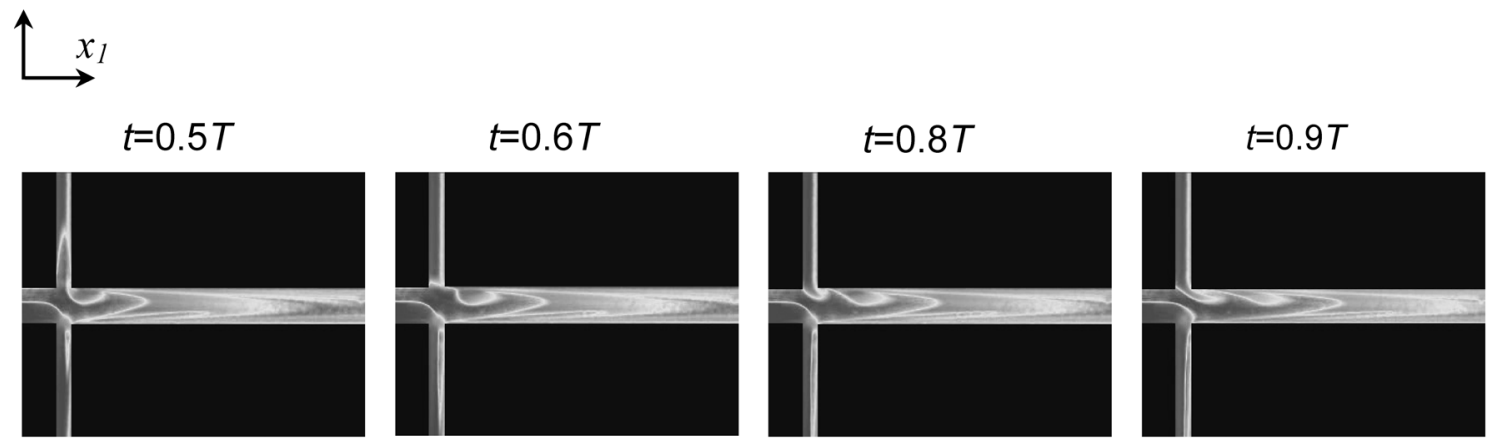

Figure 5. Pseudo-colour enhanced micrographs showing the generation of the stretching and folding of the fluids (glycerine solution with and without Rhodamine 6G dye) in the micro mixer at different stages during one period $(T=1 \mathrm{~s})$ at $\mathrm{Re}=2.4 \times 10^{-4}$ and $\mathrm{St}=0.08$. 


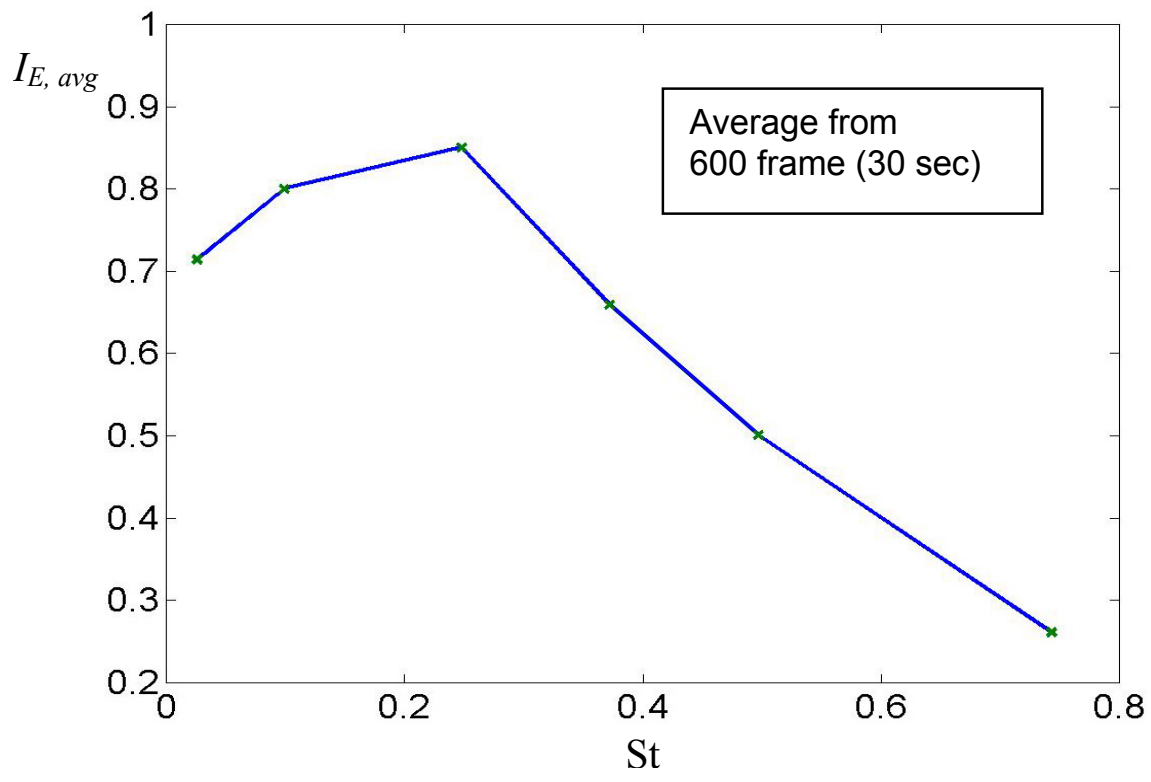

Figure 6. Time-averaged experimental mixing index as a function of Strouhal number, $\mathrm{St}$, for the simple cross-channel micro mixer at $\mathrm{Re}=$ $2.4 \times 10^{-4}$ 


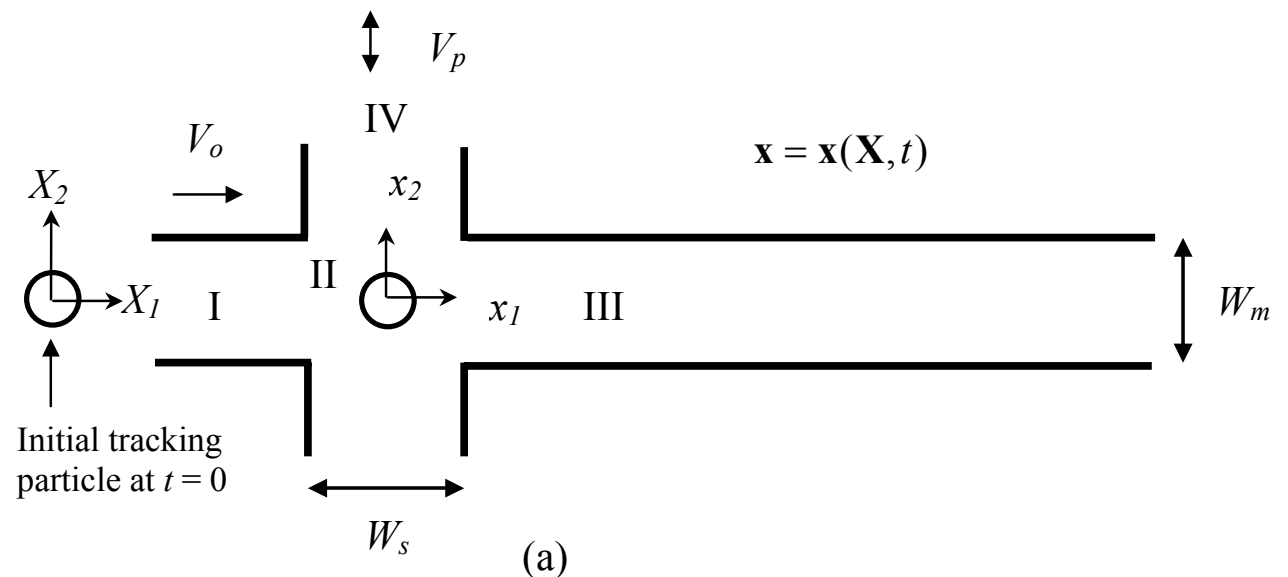

(a)

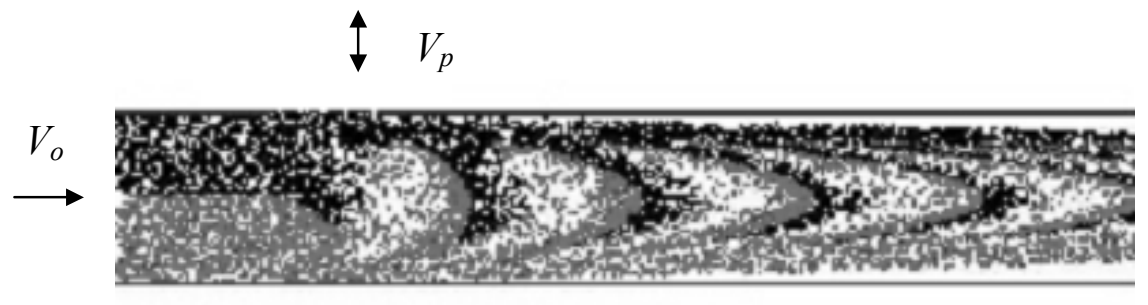

(b)

Figure 7. (a) The velocity fields in the micro mixer model divided into Regions, I, II, III and IV, (b) the generation of a lobe-like structure by the periodic perturbation of the two-stream fluids to increase the surface contact area. $X_{1}$ and $X_{2}$ are the material coordinates $\left(V_{p} / V_{0}=0.5, \mathrm{St}\right.$ $=0.637)$. 


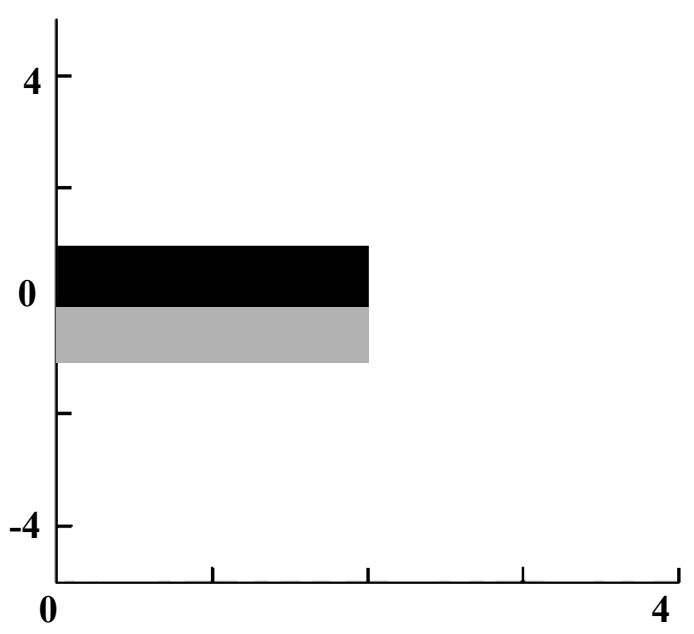

(a)

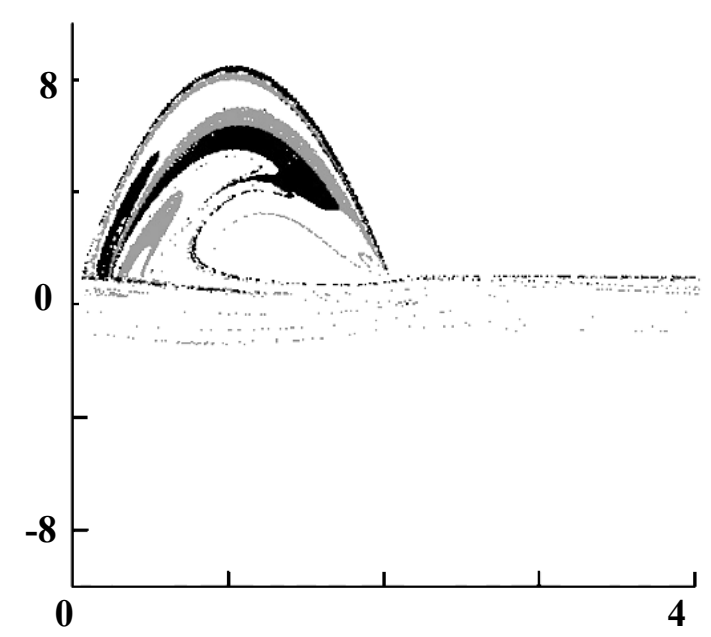

(b)

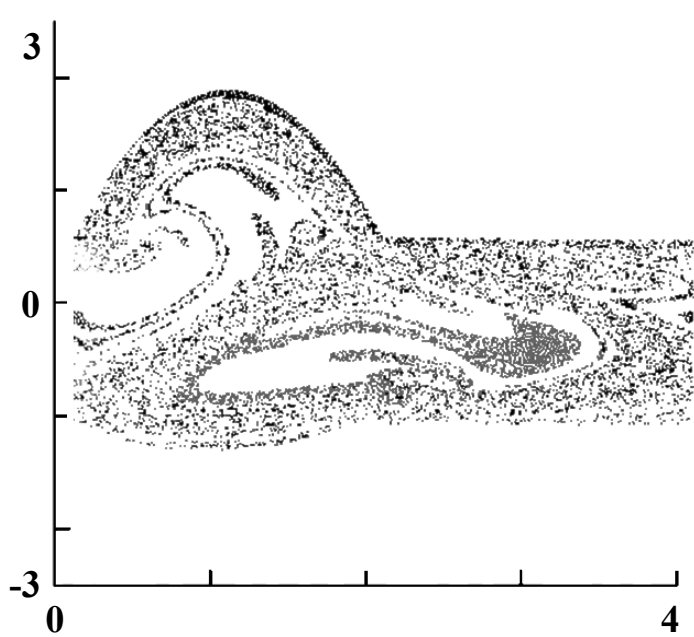

(c)

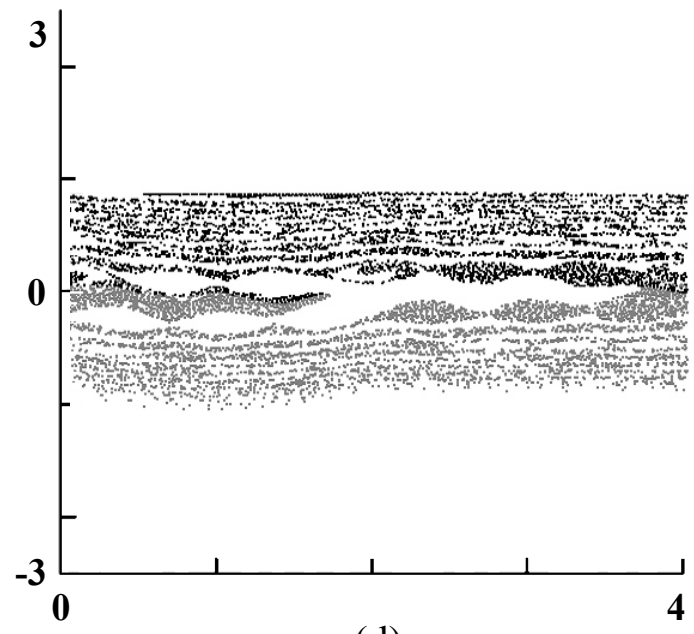

(d)

Figure 8. Simulations of mixing of two fluid blobs in the time periodic micro mixer at different driving frequencies at $t^{*}\left(=t / t_{0}\right)=13.3\left(t_{0}=d / V_{0, \text { avg }}=3\right)$ : (a) initial fluid blobs at $t^{*}=0$; (b) $\mathrm{St}=0.24, t^{*}=13.3$; (c) $\mathrm{St}=0.32, t^{*}=13.3$; and (d) $\mathrm{St}=0.95, t^{*}=13.3$. 


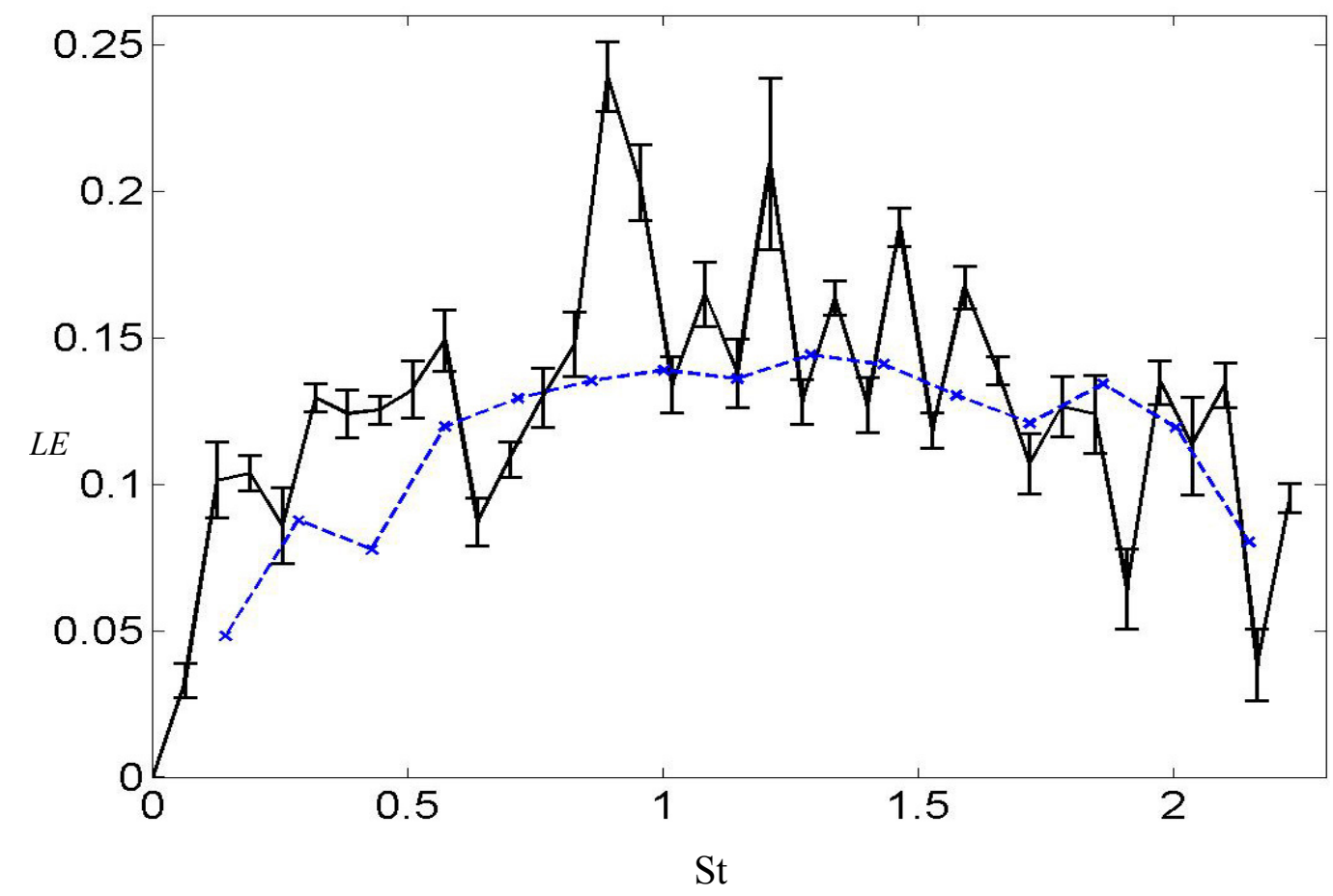

Figure 9. Comparison of the maximum Lyapunov exponent of the micro mixer model as a function of Strouhal numbers using two different methods: method I (dotted), method II (solid line with the error bars). The error bar denotes the standard deviation of the 10,000 Lyapunov exponents along the trajectory before the final time step. Initial condition $(x, y)=(0,0)$, Initial orientation $=(1,0), V_{p}=1.5$. 


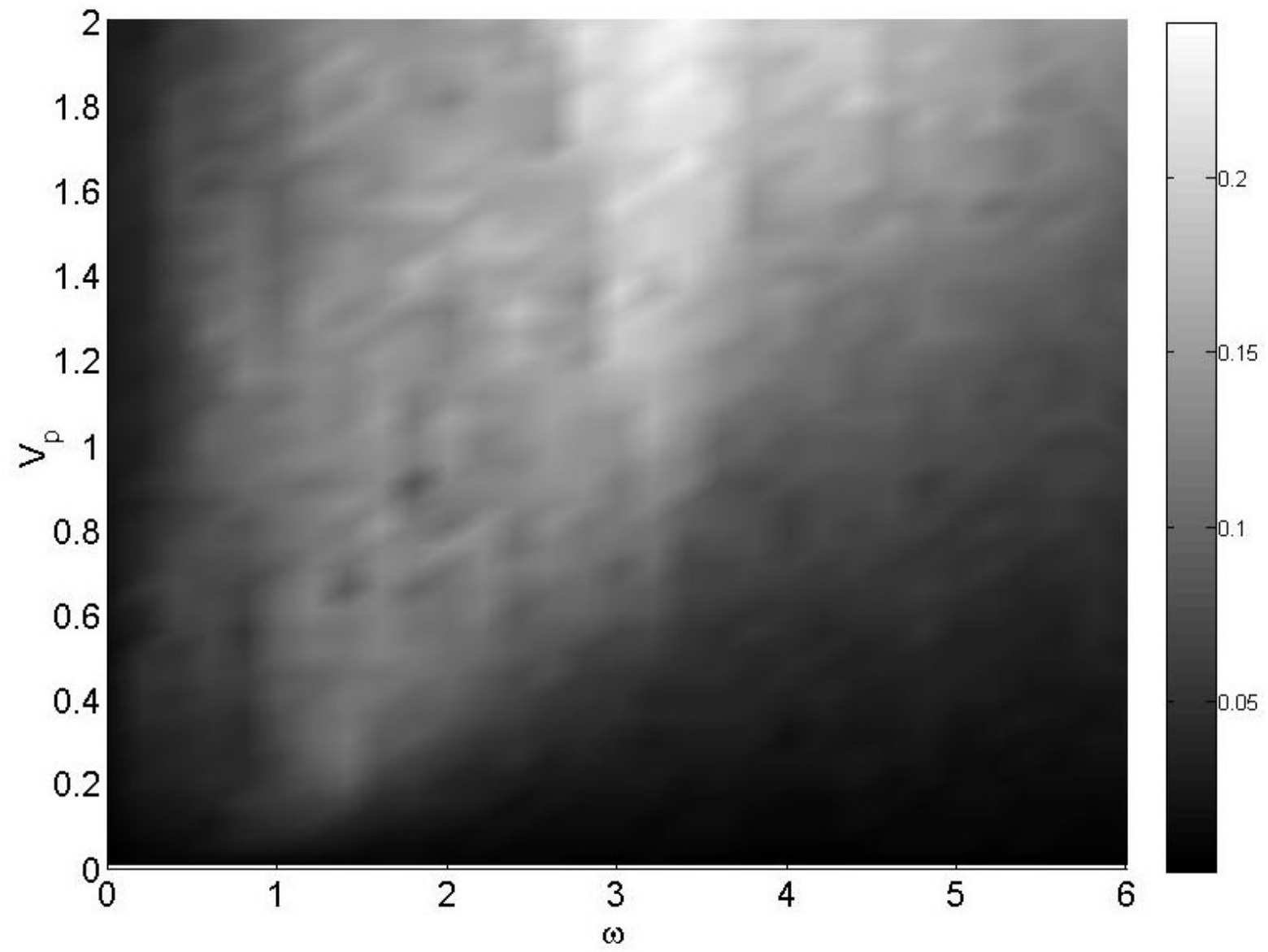

Figure 10. Contour plot of the mean Finite-Time Lypaunov exponent of the micro mixer model on the $\omega-V_{p}$ plane $\left(V_{o}=1\right)$. 


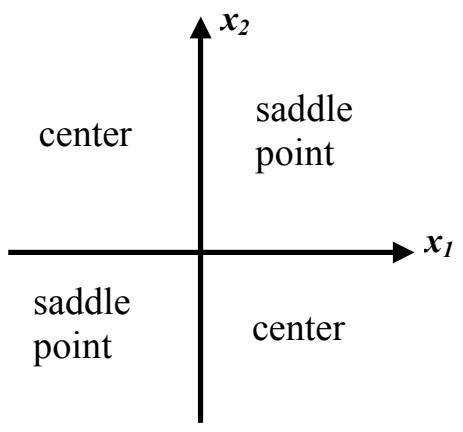

(a)

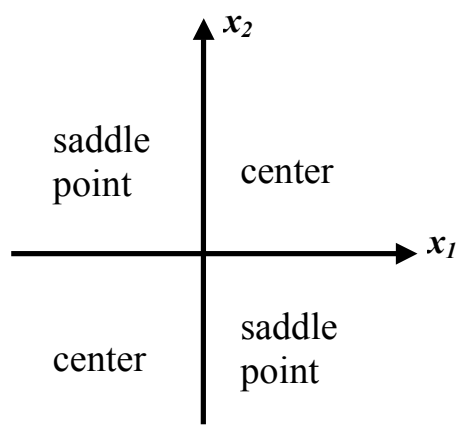

(b)

Figure 11. Eigenvalue analysis of the micro mixer model at the intersection of the main and side channel: (a) when $2 n \pi<\omega t<(2 n+1 / 2) \pi$ and $(2 n+3 / 2) \pi<\omega t<2(n+1) \pi$, and (b) when $(2 n+1 / 2) \pi<\omega t<(2 n+3 / 2) \pi(n=0,1,2, .$.$) .$ 


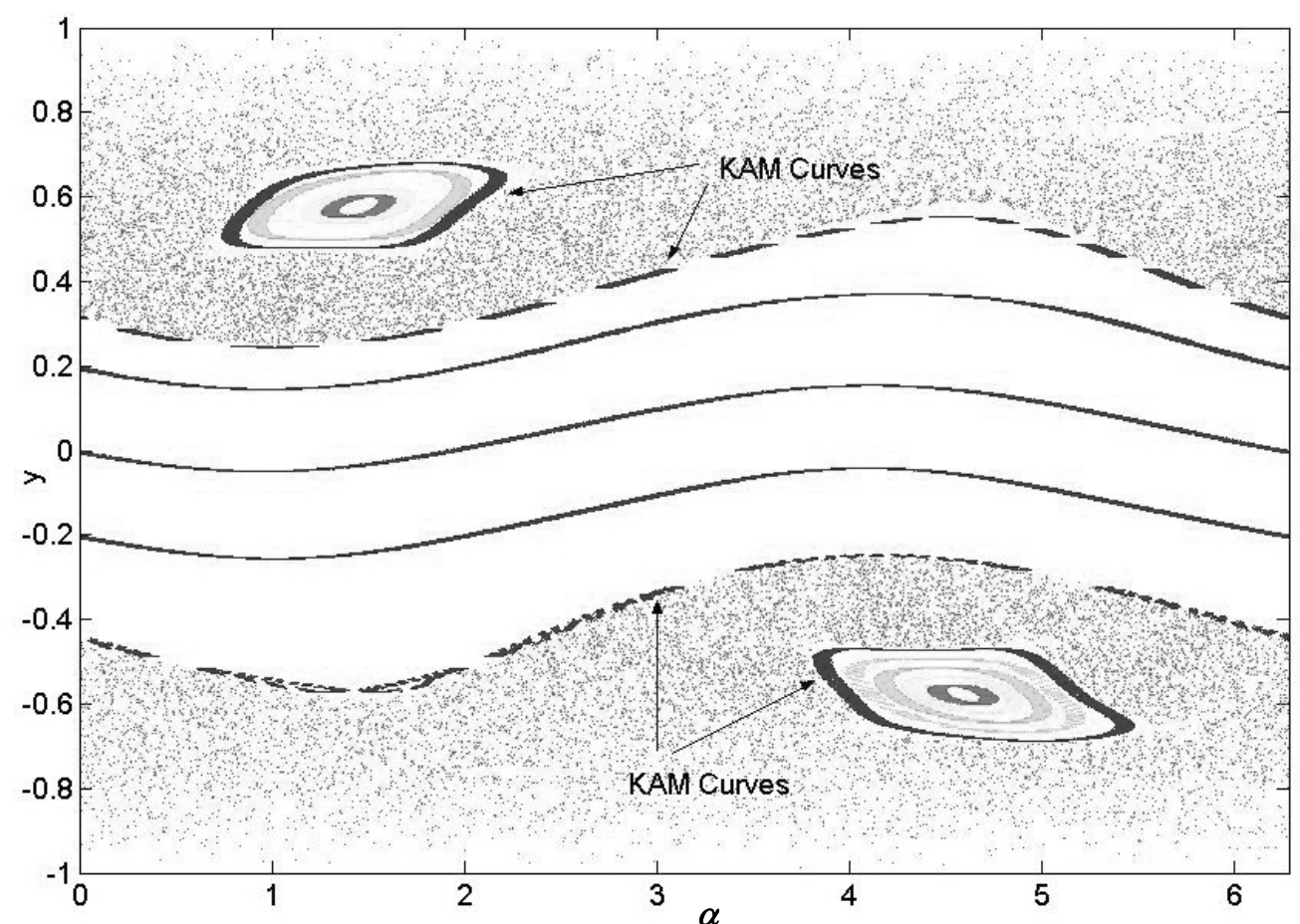

(a)

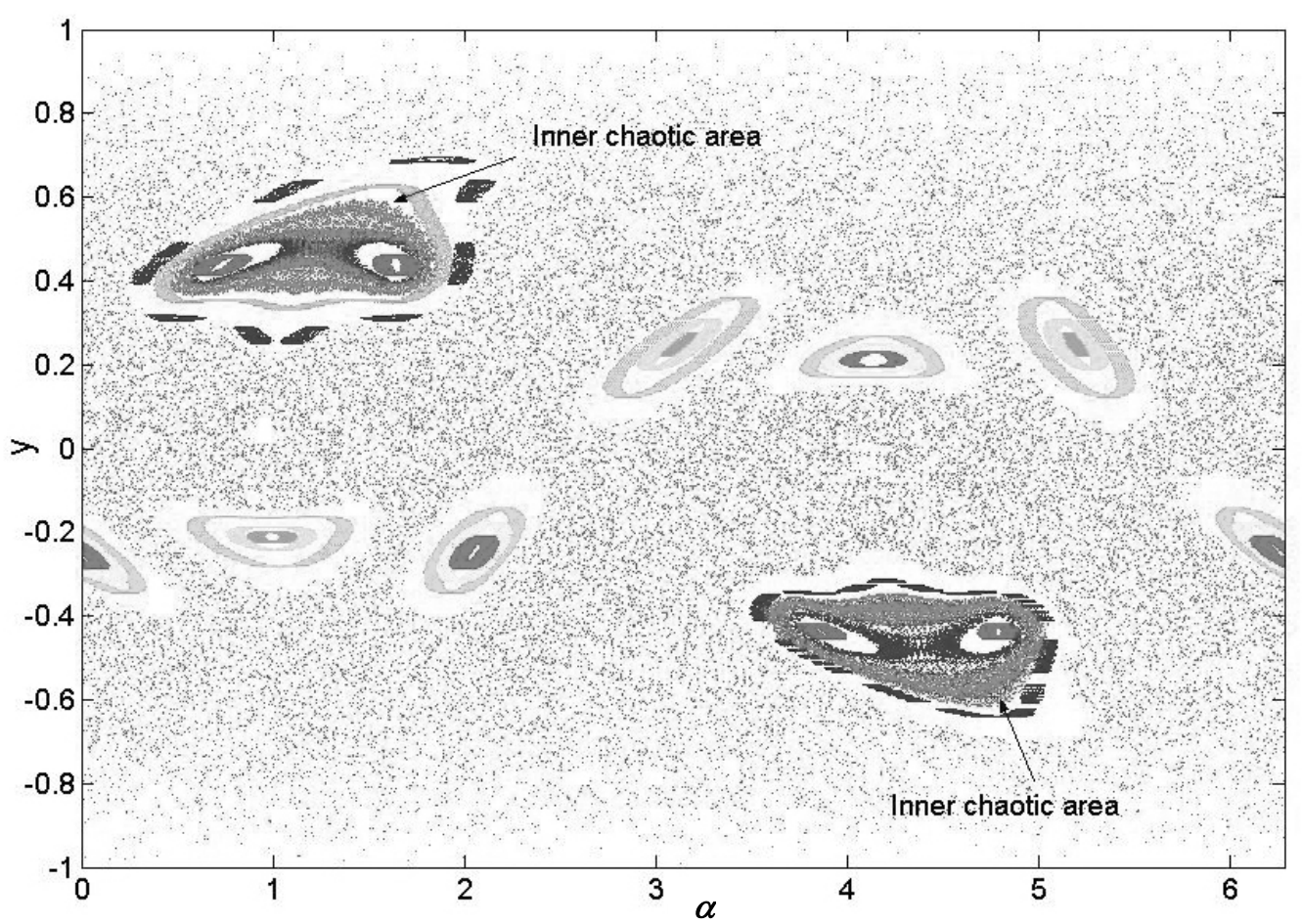

(b)

Figure 12. Poincaré sections of the micro mixer model at different conditions: (a) the two types of KAM curves formed at $V_{p}=0.15$; and (b) the train of periodic islands that appeared at $V_{p}=0.43$. 


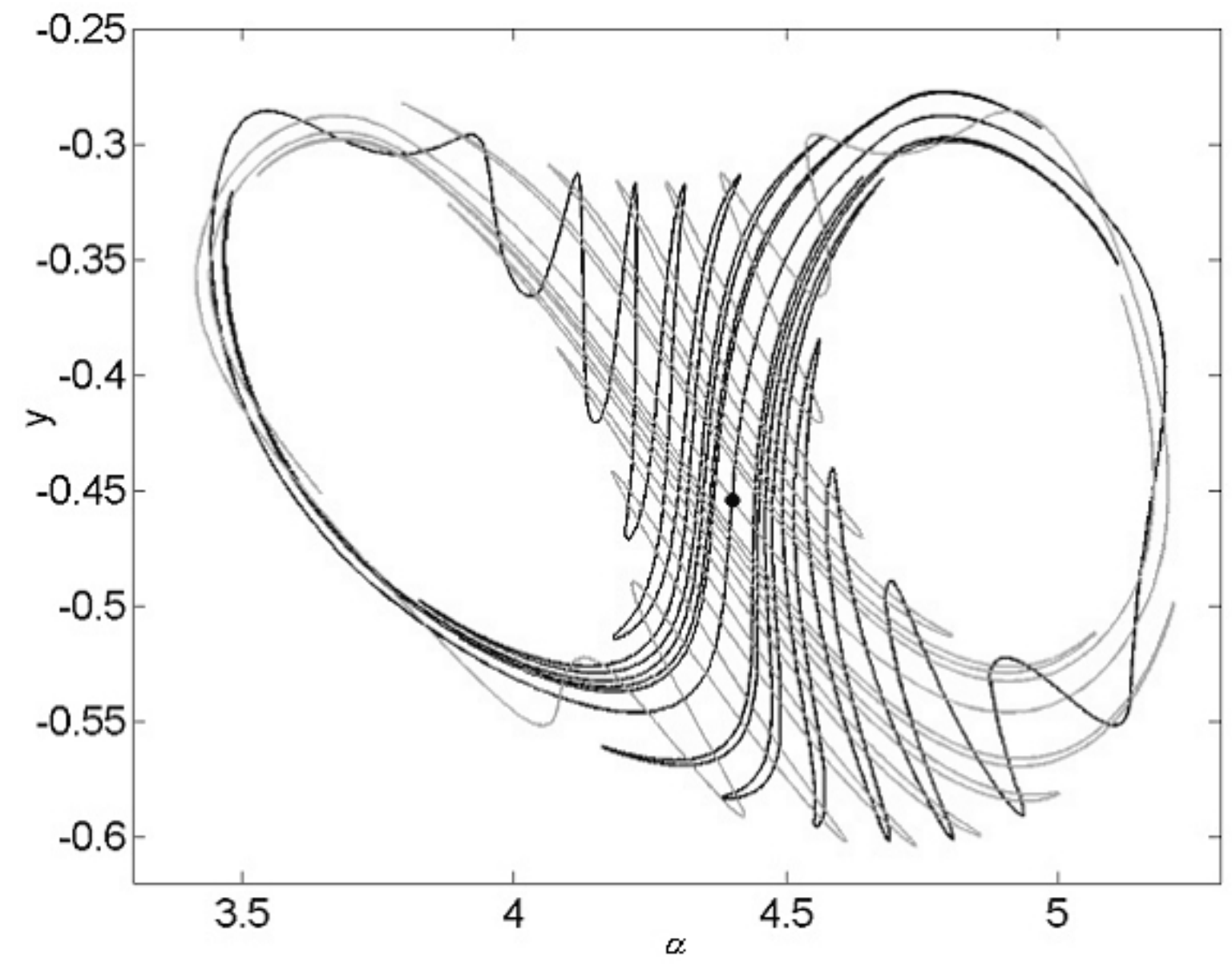

Figure 13. Stable (grey line) and unstable manifolds (black line) of a hyperbolic fixed point in the Poincare sections of the micro mixer model at $V_{p}=0.471$ and $\omega=1$. 